\title{
Investigating the electronic structure of confined multiexcitons with nonlinear spectroscopies
}

Cite as: J. Chem. Phys. 152, 104710 (2020); https://doi.org/10.1063/1.5142180

Submitted: 10 December 2019 . Accepted: 20 February 2020 . Published Online: 11 March 2020

S. Palato (D), H. Seiler (D), H. Baker, C. Sonnichsen, P. Brosseau, and P. Kambhampati (D)
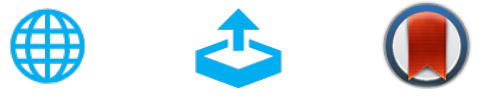

View Online

\section{ARTICLES YOU MAY BE INTERESTED IN}

Molecular polaritons for controlling chemistry with quantum optics

The Journal of Chemical Physics 152, 100902 (2020); https://doi.org/10.1063/1.5136320

Enhancement of the second harmonic signal of nonlinear crystals by self-assembled gold nanoparticles

The Journal of Chemical Physics 152, 104711 (2020); https://doi.org/10.1063/1.5139893

Comparing the excited-state properties of a mixed-cation-mixed-halide perovskite to methylammonium lead iodide

The Journal of Chemical Physics 152, 104703 (2020); https://doi.org/10.1063/1.5133021
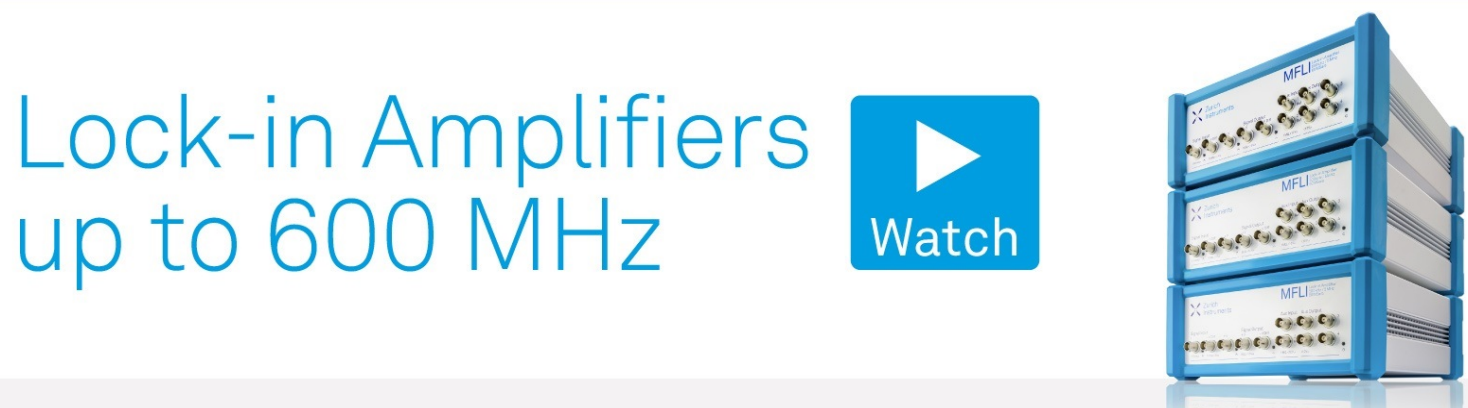


\title{
Investigating the electronic structure of confined multiexcitons with nonlinear spectroscopies
}

\author{
Cite as: J. Chem. Phys. 152, 104710 (2020); doi: 10.1063/1.5142180 \\ Submitted: 10 December 2019 - Accepted: 20 February 2020 • \\ Published Online: 11 March 2020
}

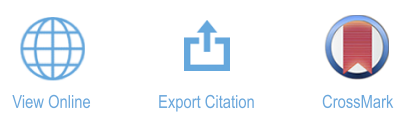

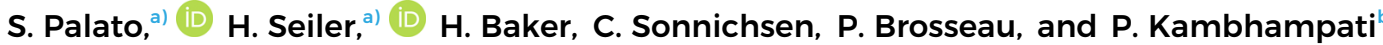

\author{
AFFILIATIONS \\ Department of Chemistry, McGill University, 801 Sherbrooke Street W, Montréal, Québec H3A OB8, Canada \\ a) Also at: Department of Physical Chemistry, Fritz Haber Institute of the Max Planck Society, Faradayweg 4-6, \\ 14195 Berlin, Germany. \\ b) Author to whom correspondence should be addressed: pat.kambhampati@mcgill.ca
}

\begin{abstract}
Strong confinement in semiconductor quantum dots enables them to host multiple electron-hole pairs or excitons. The excitons in these materials are forced to interact, resulting in quantum-confined multiexcitons (MXs). The MXs are integral to the physics of the electronic properties of these materials and impact their key properties for applications such as gain and light emission. Despite their importance, the electronic structure of MX has yet to be fully characterized. MXs have a complex electronic structure arising from quantum many-body effects, which is challenging for both experiments and theory. Here, we report on the investigation of the electronic structure of MX in colloidal CdSe QDs using time-resolved photoluminescence, state-resolved pump-probe, and two-dimensional spectroscopies. The use of varying excitation energy and intensities enables the observation of many signals from biexcitons and triexcitons. The experiments enable the study of MX structures and dynamics on time scales spanning 6 orders of magnitude and directly reveal dynamics in the biexciton manifold. These results outline the limits of the simple concept of binding energy. The methods of investigations should be applicable to reveal complex many-body physics in other nanomaterials and low-dimensional materials of interest.
\end{abstract}

Published under license by AIP Publishing. https://doi.org/10.1063/1.5142180

\section{INTRODUCTION}

One of the key features of quantum confined structures is their ability to support multiple bound states. The leading elementary excitation is an exciton, for which the physical geometry gives rise to a spectrum of states. ${ }^{1-5}$ The excitonic states and the dynamics within these states in colloidal semiconductor quantum dots (QDs) are now well established. ${ }^{6,7}$ In addition to these single excitations, nanostructures can support multiexcitons (MXs) that are also quantum confined. ${ }^{5,8-10}$ These multiexcitons are not merely insignificant corrections but can give rise to many key properties. ${ }^{11-15}$ For example, biexciton (BX) formation dynamics is central to the physics of optical gain in semiconductor quantum dots (QDs). Even in simple LED applications, the multiexciton interactions can control the performance. In short, multiexcitons are fundamental to nanostructures and confer many of their optical and electronic properties.

The biexciton, formed from two interacting electron-hole pairs, is the simplest multiexciton and has been studied in great detail. $^{14,16-18}$ The CdSe nanocrystal (NC) QD provides a model system for studying multiexciton interactions and dynamics. The simplest question is whether the biexciton (BX) is a bound or an anti-bound state. The impact of material properties such as size or composition on the biexciton binding energy is of interest both in its fundamental aspects and for applications. More deeply, one can interrogate the dominant interaction mechanism, whether the interactions are well described using mean field treatments or require the calculation of electron correlation effects. ${ }^{19,20}$ In short, MX in NCs represent a test bed of basic quantum mechanics and many-body physics.

Historically, the simplest way to observe MX was through photoluminescence (PL). ${ }^{8}$ PL experiments were critical to the initial successes in understanding the electronic properties of $\mathrm{X}, \mathrm{BX}$, and MX in epitaxial QDs. Time-resolved PL (t-PL) later provided significant advances in the field. The short lifetimes of MX in colloidal NCs posed a significant challenge to their observation via PL. Therefore, initial reports on BX in CdSe used femtosecond transient absorption (TA). ${ }^{16,21}$ Since then, a variety of NC QDs have been investigated by $\mathrm{t}-\mathrm{PL}^{8,22,23}$ and by state-resolved 
pump-probe (SRPP). ${ }^{24-27}$ Most recently, two-dimensional electronic spectroscopy (2DE) experiments have been performed on these model systems. ${ }^{28-33}$

Since MX in QD remain an interesting test bed for exploring many-body physics and for their continued relevance in new nanomaterials from perovskites ${ }^{34-41}$ to transition metal dichalcogenides, ${ }^{42-49}$ one aims for a comprehensive overview of the methods for investigation and their critical comparison. Here, we report on such an overview of the experimental signatures of MX in CdSe NCs using the three main methods: t-PL, SRPP, and 2DE. The aim of this work is to describe how different experiments reveal different processes and to what extent the results can be compared. Critically, we aim to learn from the comparison. The broader aim of this work is to provide a roadmap for future investigations using these three tools.

\section{BACKGROUND}

Quantum confinement gives rise to novel phenomena; in semiconductor nanocrystals, it progressively transforms the electronic excitations from free bulk-like charge carriers to molecular orbitals. ${ }^{1,50,51}$ CdSe NCs are typically in the strong confinement regime, where the radius of the $\mathrm{NC}$ is smaller than the Bohr radius of the exciton $a_{0}=5.6 \mathrm{~nm}$. In this regime, the elementary electronic excitation is the exciton, a bound electron-hole pair. Excitons are charge-neutral perturbations of the charge density, much like the electronic excitation of molecules. The electron and hole are delocalized over the entire volume of the NC, pinned in place by the physical boundaries of the NC and are, thus, forced to interact. Excitons are, thus, easily observed in these materials. ${ }^{2,3}$ They exhibit a rich spectrum arising from the interplay of bulklike semiconductor physics, quantum confinement, electron-hole interaction, and coupling to the lattice. ${ }^{4,9}$ Excitons in semiconductor NCs have been studied in great detail, both theoretically and experimentally. ${ }^{52-55}$

Strong confinement also enables the observation of multiexcitons (MXs) formed of multiple electron-hole pairs, which are usually not observed in the bulk. ${ }^{8,17,21,32,56}$ Due to the physical boundaries of the NC, the MXs are constrained to overlap. The interaction is further enhanced by reduced dielectric screening in low dimensions and low polarizability of the confined electrons and holes. The physics of MX in strongly confined volumes exists in a regime intermediate between bulk-like semiconductor and molecular systems. It, thus, inherits concepts from both fields. Compared to excitons, the physics of MX is influenced by electron-electron and hole-hole interactions. It is truly a quantum many-body problem. ${ }^{19}$ Due to the high-level of excitation required for their observation, the physics of MX is challenging to study theoretically. $9,19,20,57,58$

The most studied MX is the biexciton BX. MXs are usually studied by PL spectroscopy. ${ }^{8,56,59}$ This is illustrated in Fig. 1 for the case of the biexciton. Fast relaxation of the excited state ensures that PL is dominated by emission from the lowest energy states of a given manifold. The emission from BX is compared to the emission from $\mathrm{X}$, and the shift is considered equal to the biexciton binding energy $\Delta_{\mathrm{BX}}$. This simple description of the problem ignores another important feature of exciton physics: the large emission Stokes shift of $\mathrm{X}, \delta_{\mathrm{X}}$. The Stokes shift indicates the presence of a significant fine
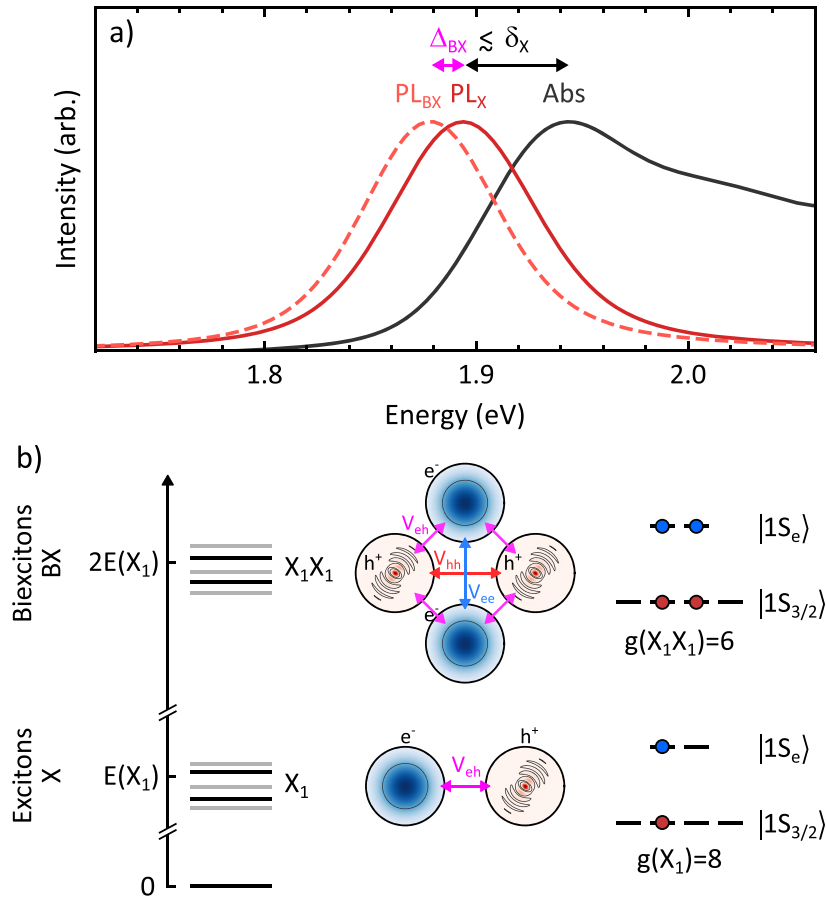

FIG. 1. Electronic structure of the biexciton BX. (a) Biexcitons are most often studied using PL experiments, revealing a well-defined shift interpreted as the binding energy $\Delta_{\mathrm{BX}}$. This simple picture disregards the electronic structure of the exciton, which is revealed by the exciton Stokes shift $\delta_{X}$. (b) The electronic structure of strongly confined semiconductor NCs can be represented in the exciton basis (left) or the electron-hole basis (right). The exciton basis includes the interactions between electrons and holes (middle). The degeneracy of the electron-hole configurations is indicated on the right panel.

structure in the band-edge exciton manifold. There must be at least two $\mathrm{X}_{1}$ states: an emissive state favored in emission experiments and an absorptive state favored in absorption experiments. PL lifetime is also significantly influenced by this fine structure. ${ }^{2,60}$ Furthermore, the Stokes shift is typically comparable to or larger than the biexciton shift. There are, thus, multiple final states for emission from the biexciton. There is no guarantee that $P L_{X}$ and $P L_{B X}$ involve the same state or even that $\mathrm{PL}_{\mathrm{BX}}$ involves an exciton for which the transition to the ground state is allowed. The interpretation shown in Fig. 1 (a) requires that the final state for $\mathrm{X}_{1} \mathrm{X}_{1}$ emission is also the emissive $\mathrm{X}_{1}$ state. If, instead, $\mathrm{X}_{1} \mathrm{X}_{1}$ emission is considered to yield the absorptive $\mathrm{X}_{1}$ state, the binding energy would be around $-30 \mathrm{meV}$. The situation is, thus, not as clear as the simple PL signals might suggest.

The difficulties arising from measuring the energetics of multiexcitons are further compounded when considering their complex electronic structure. The electronic structure of a semiconductor NC is illustrated in Fig. 1(b) in the exciton basis (left) and in the electron-hole basis (right), according to effective mass models. ${ }^{9}$ The exciton basis can be obtained from the electron-hole basis when including interactions between the electrons and holes (middle panel). Although the electron-hole basis is not an eigenstate of the system, it can be used to derive the properties of the excitons such as 
the number of states in the manifold. The band-edge exciton $\mathrm{X}_{1}$ in CdSe NCs is formed by electrons in the 2 -fold degenerate $1 S_{e}$ state and holes in the 4 -fold degenerate $1 S_{3 / 2}$ state. For the single exciton, there are, thus, eight possible configurations. The biexciton requires two electron-hole pairs, which saturates the electron level. There are six possible configurations, corresponding to the possible arrangement of the two indistinguishable holes in four levels. These simple considerations immediately predict a fine structure for the biexciton, which arises from the same many-body effects as the exciton fine structure and biexciton binding.

The current work wishes to discuss the electronic structure of multiexcitons. We designate exciton states $\mathrm{X}_{i}$, where $i$ is a phenomenological label used for distinguishable spectroscopic transitions. This is often called the coarse structure of the exciton. The lowest exciton, $\mathrm{X}_{1}$, will be called the band-edge exciton, following common usage. Biexcitons will be designated as BX. When multiple transitions can be identified, they will be indicated as indices. The biexcitons generated from the band-edge exciton by the excitation of a second electron-hole pair will be labeled $\mathrm{X}_{1} \mathrm{X}_{i}$. The lowest biexciton, $\mathrm{X}_{1} \mathrm{X}_{1}$, will similarly be called the band-edge biexciton. The binding energy of a given biexciton can be observed using both absorptive $\left(\Delta_{\mathrm{Abs}}\right)$ and emissive $\left(\Delta_{\mathrm{Em}}\right)$ experiments. The biexciton will be indicated by the argument, for example, $\Delta_{\mathrm{Abs}}\left(\mathrm{X}_{i} \mathrm{X}_{j}\right)$. The binding energy will be defined as the difference between the energy of the biexciton and the sum of the energy of the constituent excitons, $\Delta\left(\mathrm{X}_{i} \mathrm{X}_{j}\right)=E\left(\mathrm{X}_{i} \mathrm{X}_{j}\right)-E\left(\mathrm{X}_{i}\right)-E\left(\mathrm{X}_{j}\right)$. The binding energy of the triexciton (TX) will similarly be defined as the difference between the energy of the triexciton and the sum of the energy of the corresponding single excitons. The fine structure states will not be labeled individually as they cannot be resolved experimentally in the current ensemble measurements at room temperature. Instead, the difference between the absorptive and emissive transitions will be used as an indication of the widths of the exciton manifolds. The Stokes shifts of the band-edge exciton, biexciton, and triexciton will, respectively, be labeled $\delta_{\mathrm{X}}, \delta_{\mathrm{BX}}$, and $\delta_{\mathrm{TX}}$.

Excitons in NCs can be described in two bases: the electronhole basis and the exciton basis. Although both bases are valid choices, some phenomena can be expressed or interpreted more directly in one basis or the other. The electron-hole basis is closely related to semiconductor physics. It represents the electron and hole as two particles in their own manifolds with their own dynamics. According to the effective mass model of semiconductor NCs, the electron and hole states are obtained from the bulk bands upon confinement. ${ }^{4,20,61}$ Although they correspond directly to the continuous bands of semiconductors physics, they form discrete states similar to molecular orbitals. The electron and hole states are single particle states obtained in the absence of electron-hole interaction, and they are, thus, not the eigenstates of the system. The inclusion of electron-hole interaction gives rise to the exciton states, interacting electron-hole pairs. They are, thus, the elementary electronic excitations in these materials and form the basis of a rigorous discussion of the optical response. The exciton basis, containing both $\mathrm{X}$ and $\mathrm{MX}$, describes the electronic state of the entire nanocrystal: it is the state of the entire system. Due to confinement, there cannot be two non-interacting excitons in the same NC. Of course, the exciton states can be obtained from a linear combination of electron-hole states. Since both are valid choices, the choice of basis varies depending on the phenomenon of interest.

Although the exciton basis is the eigenbasis of the system, the excitons can be a bit abstract. The electron-hole basis will, thus, be frequently invoked to explain specific phenomena. Indeed, the electron-hole basis can be used as a complete basis set for the calculation of exciton wavefunctions. ${ }^{4,20}$ Many properties of the electronhole states are conserved when transferring to the exciton basis. The electron-hole basis is, thus, a useful tool to compute the properties of specific excitons. For example, the number of states in $\mathrm{X}_{1}$ and $\mathrm{X}_{1} \mathrm{X}_{1}$ manifolds were previously determined from the degeneracies of the electron and hole states [Fig. 1(b)]. Surface trapping and Auger processes are also more easily discussed using independent electrons and holes as is dot charging. The electron-hole basis will, thus, be used when discussing specific population dynamics.

The exciton basis is the appropriate basis when discussing the spectroscopic properties of the NC. Essentially, all derivations of light-matter interaction start by separating the Hamiltonian into a material part and a light-matter interaction part and then use the material part as a reference Hamiltonian to define the interaction picture. ${ }^{62-65}$ As a result, the spectroscopic transitions directly connect the eigenstates of the material Hamiltonian, the exciton states. This is a subtle but an important distinction. Many experimental spectroscopic studies on CdSe QDs discuss the spectroscopic transitions in the electron-hole basis. ${ }^{10}$ In the process, they make it difficult to account for the effect of electron-hole interactions, the salient physics of the system. ${ }^{66}$ We will discuss the transitions as occurring between exciton states. The electron-hole basis will, nevertheless, be useful when discussing the properties and dynamics of the exciton states, such as number of states, approximate transition dipole moments, and dynamics. Furthermore, we wish to emphasize the difference between ground and excited states. In semiconductor QDs, the ground state $(G)$ is the exciton vacuum, and it can only exhibit vibrational dynamics (or recovery following excitation). Holes are part of the excited state and, thus, can only be discussed in connection with excited state signals: stimulated emission (SE) and excited state absorption (ESA). The basis of excitons should be used when discussing absorption, whether from the ground or excited states, and emission, whether spontaneous or stimulated.

The properties of excitons have been computed using multiple levels of theories from effective particle-in-a-box treatments to full $a b$ initio calculations. The above discussion follows the logic and results of the multiband effective mass approximation (EMA) model of NCs. ${ }^{4,9,61}$ In this model, the bands of the bulk semiconductor are used to obtain the single-particle wavefunctions for the electrons and holes in a confining potential. The electron and hole states are used to obtain the exciton states after inclusion of electronhole interaction. The structure of the exciton and multiexcitons arises from the NC size and shape, lattice symmetry, and the interactions between electrons and holes. The EMA model of NCs is the standard model used when discussing the electronic structure of semiconductor NCs. Higher levels of theory have been applied to the electronic structure of NCs. Importantly, atomistic methods enable the computation of the electronic structure for realistic NCs. ${ }^{20,52,55,67-69}$ In general, the atomistic methods break the symmetry that is present in continuum models, lifting degeneracies and further mixing states and, thus, giving rise to a more complicated 
manifold of fine-structure states. Nevertheless, the multiple theoretical treatments at different levels of theory generally agree on the properties of the first few excitons.

The electronic structure of multiexcitons has been subject to significant theoretical work. It is, indeed, an exciting problem containing an important dilemma: accurate treatment of the quantum mechanical many-body aspects is only feasible when using approximations of the NC structure. This challenge has motivated investigations at multiple levels of theories from quasi-analytical to atomistic. ${ }^{9,19,20,57,66,70}$ All models predict a dense manifold for the biexciton arising from the interplay of direct and exchange Coulomb interaction. The models agree on the dominant role of exchange interactions in determining the energy and level structure. Again, the atomistic nature of the NC breaks the symmetry of the problem, generally resulting in the lifting of degeneracies, giving rise to further mixing of the states. The electronic structure of the multiexciton is as rich, if not more, as the single excitons.

The experimental studies of MX require non-linear spectroscopy, which is sensitive to multiply excited states. Three timeresolved experiments are used, as shown in Fig. 2. All experiments use a strong excitation pulse to generate $\mathrm{X}$ or $\mathrm{MX}$ populations. The signal depends on the properties of the exciting pulse. We will
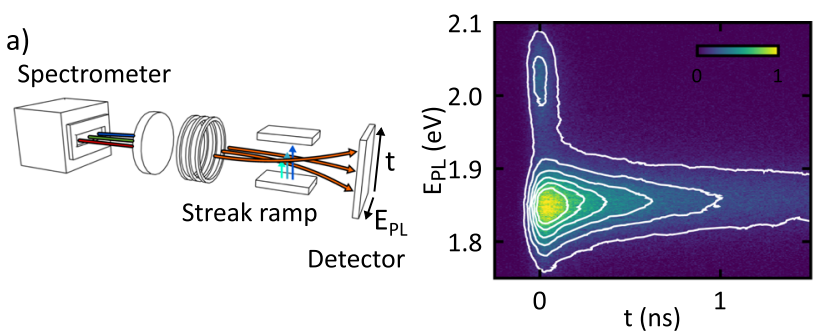

b)

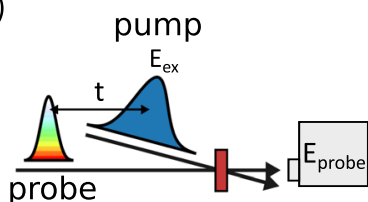

c)
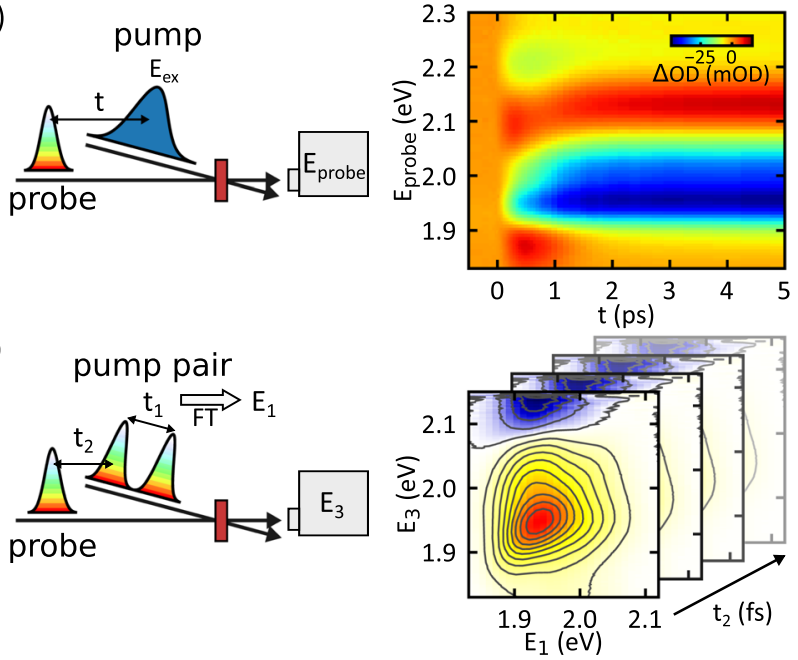

FIG. 2. Non-linear spectroscopy experiments. (a) Time-resolved photoluminescence (t-PL) using a Streak camera resolves the emission spectrum on time scales from $100 \mathrm{ps}$ to $1 \mu \mathrm{s}$. (b) State-resolved pump-probe (SRPP) enables the study of absorption and emission from excited NC populations. (c) Two-dimensional electronic (2DE) spectroscopy reveals the spectroscopic response as a function of excitation energy $\left(E_{1}\right)$, detection energy $\left(E_{3}\right)$, and pump-probe delay $t_{2}$. refer to the energy of the exciting photon $E_{\mathrm{ex}}=h c / \lambda$ as the excitation energy, in $\mathrm{eV}$. The excitation intensity $I_{0}$ refers to the energy content per pulse, in $\mathrm{nJ} /$ pulse. The three experiments differ in the information they reveal. Time-resolved PL (t-PL) measures the PL intensity as a function of both emission time $t$ and energy of the emitted photon $E_{\mathrm{PL}}$. State-resolved pump-probe (SRPP) measures the change in optical density $(\Delta \mathrm{OD})$ as a function of pump-probe delay $t$ and probe energy $E=h c / \lambda$. Two-dimensional electronic spectroscopy is related to SRPP. However, it measures a change in the transmission as a function of three axes: excitation energy $E_{1}$, pump-probe delay $t_{2}$, and probe energy $E_{3}$. In $2 \mathrm{DE}$, the excitation is performed by two pulses; therefore, it is equivalent to a TA experiment carried out with twice the intensity. The three techniques have different advantages in revealing the electronic structure of multiexcitons.

\section{METHODS}

\section{A. Samples}

The experiments focus on a single size and type of QD. Wurtzite CdSe QD with octadecylamine ligands dissolved in toluene was obtained commercially (NNLabs, CSE-640). Their band-edge absorption was $640 \mathrm{~nm}$, with a typical quantum yield of $\sim 40 \%$. All spectroscopic experiments were carried out while flowing the sample using a peristaltic pump (Masterflex 77390-00). A total of three different batches were used throughout the paper.

\section{B. Time-resolved photoluminescence}

The t-PL spectra are acquired using a streak camera (Axis TRS, Axis Photonique Inc.). A femtosecond pulse excites the sample. The $\mathrm{PL}$ is collected at a $90^{\circ}$ angle and imaged to the slit at the entrance of the streak camera using a pair of off-axis parabolic mirrors. The principle of operation of the streak camera is illustrated in Fig. 2(a). The streak camera has three components: a grating monochromator, a streak tube, and a detector. The spectrum of the PL is resolved by a spectrometer (Acton SP-2358i, $150 \mathrm{~g} / \mathrm{mm}, 600 \mathrm{~nm}$ blaze), thus encoding the wavelength in the horizontal position. The spectrum is converted to electrons using a photocathode, followed by acceleration of the electrons in a bilamellar streak tube (Photonis P820). During the propagation through the tube, the electrons are subjected to a time-dependent high voltage streak ramp, thus converting the arrival time into the vertical position. The streak ramp is triggered electronically using a synchronization output of the laser amplifier and a delay generator (DG645, Stanford Research Systems). Finally, the electrons are detected using a phosphor and CCD (Spectral Instruments 1200 series). The resulting image resolves the emitted spectrum in both energy, along the horizontal axis, and time, along the vertical axis. The resulting spectral resolution is about $0.2 \mathrm{~nm}$. The temporal resolution depends on the settings of the high-voltage ramp. Two ramps are used in the current work: a fast ramp [temporal range: $\sim 10 \mathrm{~ns}$, instrument response function (IRF): 120 ps FWHM] and a slow ramp (temporal range: $\sim 1 \mu \mathrm{s}$, IRF: $2.2 \mathrm{~ns}$ FWHM).

Experiments with $I_{0} \leq 50 \mathrm{~nJ} / \mathrm{p}$ averaged 20 images acquired with 15 s exposure time each; experiments with $75 \leq I_{0} \leq 250$ averaged 10 images acquired with $15 \mathrm{~s}$ exposure time; experiments with $I_{0} \geq 500 \mathrm{~nJ} / \mathrm{p}$ used 10 images acquired with $10 \mathrm{~s}$ exposure time. 
A background image is acquired before each image with the excitation beam blocked. This image is then used for background subtraction. The final traces are corrected for electrode and detector alignment (1\% shear) and the non-constant time and energy bin widths.

The sample (NNLabs CSE-640) is diluted with toluene to an OD of 0.2 at $640 \mathrm{~nm}$ in a $0.5 \mathrm{~mm}$ path length cell. During the experiment, this sample is flowed in a $0.5 \mathrm{~mm}$ path length flow cell (Starna Type 48). Excitation pulses at $400 \mathrm{~nm}$ and $640 \mathrm{~nm}$ in the range of $1-1000 \mathrm{~nJ} /$ pulse with a duration of $<100$ fs are generated by coupling the output of a Ti:sapphire regenerative amplifier (Coherent Legend Elite Duo HE+, $1 \mathrm{kHz}$ rep rate) to either a $100 \mu \mathrm{m}$ thick BBO crystal (frequency doubling to obtain $400 \mathrm{~nm}$ ) or to an optical parametric amplifier (OPA, OPerA Solo, Coherent) to obtain $640 \mathrm{~nm}$ light.

\section{State-resolved pump-probe}

The ultrafast SRPP instrument used here has been described previously. ${ }^{71}$ Briefly, the output of a Ti:Sapphire amplifier (Coherent Legend USP, $820 \mathrm{~nm}, 1 \mathrm{kHz}, 70 \mathrm{fs}, 2.2 \mathrm{~mJ} /$ pulse) is split into three arms. Two of those arms (approximately $1 \mathrm{~mJ}$ each) pump two optical parametric amplifiers (OPAs) for color conversion. The third arm is used to create the white light continuum probe, and in the case of $3.1 \mathrm{eV}$ pumping, used for second harmonic generation to create the $410 \mathrm{~nm}$ pump. Each OPA output is compressed using individual prism compressors. The white light probe is generated through self-phase modulation in a $1 \mathrm{~mm}$ sapphire window. The probe is compressed using a prism compressor. After transmission through the sample, the probe is passed through a monochromator and detected with a photodiode. TA spectra are recorded by scanning the monochromator at a fixed pump-probe delay.

The chirp of the white light probe is characterized through sum-frequency generation (SFG) with the $800 \mathrm{~nm}$ fundamental at the sample position. The chirp is removed in situ by moving the delay stages in accordance with the measured chirp as the probe wavelength is scanned.

Two pump-probe experiments are performed in parallel by using two pump beams, each chopped at $333 \mathrm{~Hz}$. The choppers are phased such that over three laser shots, the sample is pumped by one pump, followed by the second, then not pumped at all. The sample is probed at each laser shot. The TA signal is measured by comparing the transmission of the probe when the pump is absent to the transmission of the probe following each pump. Each pump arm has its own computer-controlled delay stage.

The duration of the fundamental and OPA laser pulses are determined using autocorrelation in BBO. They are each under $80 \mathrm{fs}$. The IRF is determined through SFG between the fundamental pulse and the white light continuum in BBO. The IRF was found to be less than $100 \mathrm{fs}$.

\section{Two-dimensional electronic spectroscopy}

For the two-dimensional electronic (2DE) spectroscopy measurements, we employ the pulse-shaper based instrument described in detail in our recent works. ${ }^{33,72}$ Roughly, $600 \mu \mathrm{J}$ pulses centered at $800 \mathrm{~nm}$ (Coherent Legend Elite Duo HE+, $1 \mathrm{kHz}$ rep rate, $<130 \mathrm{fs}$ pulse duration) are focused into a hollow-core fiber (HCF) filled with argon in a differential-pressure setting. The input pulse is broadened via self-phase modulation by propagating through the fiber. At the output of the fiber, a shortpass filter is used to select the visible part of the continuum, yielding $40 \mu \mathrm{J}$ pulses in the 540-700 nm spectral range. The visible pulses are then collimated and sent to GRISMs (GRatings + prISMs) to pre-compensate for the large dispersion arising from acousto-optic pulse shapers (Dazzler, Fastlite), where the 2DE pulse sequences are generated. The beam is split into two arms, a pump arm and a probe arm, each equipped with a pulse shaper. In the pump arm, the pulse-shaper is employed to create a pair of compressed phase-locked pulses with controllable delay $t_{1}$ and relative phases. In the probe arm, the pulse-shaper is used to create the compressed probe pulse with pump-probe delay $t_{2}$. The spectral phase of the pulses is optimized by iteratively applying a phase mask in the pulse-shaper and characterized in a dispersion-free line using transient-grating frequency-resolved optical gating (TG-FROG). ${ }^{73}$ Typical retrieved pulse durations are $11-15 \mathrm{fs}$.

The pump and probe beams are focused and crossed in the sample. The spectrum of the transmitted probe beam is detected using a spectrometer (Acton 2500i + PIXIS 100B Excelon). In this pumpprobe implementation of $2 \mathrm{DE}$, both rephasing and non-rephasing 2DE signals are emitted in the same direction and the probe beam also acts as the local oscillator, thus yielding an automatically phased 2DE absorptive spectrum. Unwanted signals are eliminated using a $4 \times 1 \times 1$ phase-cycling procedure since standard pump-probe and linear signals do not depend on the constant phase difference between the two pumps. ${ }^{74}$ Delay $t_{1}$ is sampled from 0 fs to $100 \mathrm{fs}$ in steps of $1.0 \mathrm{fs}$. The acquisition is performed using a rotating frame with frequency $307.45 \mathrm{THz}$. Reference spectra are interleaved throughout the dataset, and they are used to normalize the signal. The sample is diluted with toluene to an OD of 0.3 at $640 \mathrm{~nm}$ in a $0.2 \mathrm{~mm}$ path length cell (Starna) and flowed using a peristaltic pump.

\section{RESULTS}

\section{A. Static spectroscopic properties}

The energy spectrum of strongly confined semiconductor NCs can be described as a progression of quantized excitons. Figure 3(a) shows the exciton manifold in the electron-hole basis (left) and exciton basis (right), with exciton states labeled $\mathrm{X}_{i}$. The electronhole basis consists of single-particle wavefunctions in the absence of electron-hole interactions. ${ }^{4,61}$ Taking into account the Coulombic interaction between the electron and hole gives rise to a manifold of excitons, which is the eigenbasis of the QD. Although some mixing of the single-particle states can occur, it remains limited for the lowest excitations. The correspondence can, thus, be made between specific electron-hole pairs and exciton states. For example, the $\mathrm{X}_{1}$ exciton consists of a $1 \mathrm{~S}_{e}$ electron and a $1 \mathrm{~S}_{3 / 2}$ hole. The correspondence is indicated by the color-coded arrows in both diagrams.

The single exciton $\mathrm{X}_{1}$ has eight possible electron-hole configurations. The degeneracy among these is lifted due to exchange interactions, crystal field splitting, and the details of the shape of the nanocrystal. ${ }^{4,9,69,75-77}$ These perturbations give rise to a finestructure for every exciton, represented in Fig. 3(b). The details 
a)

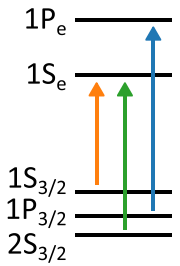

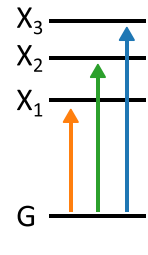

b)
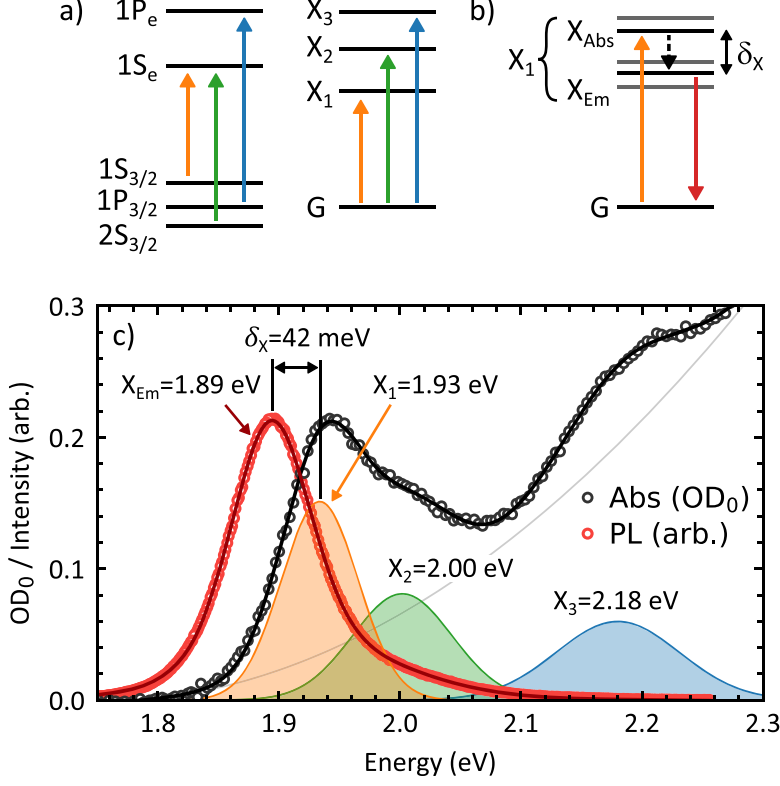

FIG. 3. Linear optical properties of excitons in CdSe NCs reveal the electronic structure of the exciton. (a) The coarse electronic structure can be expressed either in an electron-hole (left) or exciton basis (right). (b) Each of these exciton states $X_{i}$ possesses a fine structure. The PL Stokes shift can be explained by different absorptive and emissive states, separated by energy $\delta x$. (c) Linear absorption and PL spectra of CdSe NCs. The absorption spectrum can be decomposed into a series of peaks, corresponding to different exciton transitions.

of the fine-structure vary between models and depend on model parameters. For spectroscopic purposes, a minimum of two states can be assumed: an absorptive state $\mathrm{X}_{\mathrm{Abs}}$ and an emissive state $\mathrm{X}_{\mathrm{Em}}$. The absorptive state is the preferred state for light-induced absorption due to its larger absorption cross section, the combination of oscillator density of states and transition dipole moment. The emissive state is the preferred state for emission following equilibration in the fine-structure manifold. This difference between the states preferred for absorption and those preferred for emission will be invoked repeatedly throughout the paper. We label the shift between these two transitions as $\delta_{\mathrm{X}}$. The Stokes shift contains contributions from shifts between the electronic states and reorganization of the NC lattice and solvent. In CdSe NCs, the latter effects are considered to contribute $<5 \mathrm{meV},{ }^{78,79}$ such that the Stokes shift is mostly due to shifts in the electronic populations. The experimental determination of the Stokes shift, thus, provides an experimental estimate of the width of the exciton manifold.

The linear absorption and PL spectra of CdSe NCs reveal the quantized nature of excitons, as shown in Fig. 3(c). The PL spectrum (red symbols) reveals a single transition well described using a single Voigt function (red line), yielding a central emission energy of $1.892 \mathrm{eV}$. The absorption spectrum (black symbols) reveals a progression of absorption features, corresponding to the different single exciton states. The transition energies can be obtained by modeling the linear absorption spectrum, $\mathrm{OD}(E)$, following a previous procedure introduced by Zhang. ${ }^{80}$ The model, expressed in Eq. (1), is a series of three Gaussians. A quadratic polynomial is added to remove the unresolved background,

$$
\begin{gathered}
\mathrm{OD}(E)=\sum_{i=1}^{3} \alpha_{i} G\left(E, E_{i}, \sigma_{i}\right)+b_{0}+b_{1} E+b_{2} E^{2}, \\
G\left(E, E_{i}, \sigma_{i}\right)=\exp \left[-\frac{1}{2}\left(\frac{E-E_{i}}{\sigma_{i}}\right)^{2}\right],
\end{gathered}
$$

where $\alpha_{i}$ is the amplitude, proportional to the absolute square of the transition dipole moment of each transition, $\sigma_{i}$ is the Gaussian linewidth, and $E_{i}$ is the central energy. The resulting energies are $E\left(\mathrm{X}_{1}\right)=1.934 \mathrm{eV}, E\left(\mathrm{X}_{2}\right)=2.002 \mathrm{eV}$, and $E\left(\mathrm{X}_{3}\right)=2.180 \mathrm{eV}$. The resulting Stokes shift is $\delta_{\mathrm{X}}=42 \mathrm{meV}$. For reference, a direct comparison of the maximum position for $\mathrm{PL}$ and first absorption feature yields a value of $49 \mathrm{meV}$.

Linear spectroscopy allows the characterization of the single exciton manifold. It reveals the electronic structure of excitons. Separable signatures of the different exciton states $\mathrm{X}_{i}$ are readily observed. The large Stokes shift of the band-edge exciton indicates the presence of a significant fine structure, in agreement with theoretical calculations. ${ }^{4,60,61}$ However, linear spectroscopy does not enable the study of the MX. For further investigations, non-linear, time-resolved spectroscopies are needed.

\section{B. Time-resolved photoluminescence}

Time-resolved PL enables the observation of short-lived populations that cannot be observed in static PL. PL is the principal tool used for the observation of excitons and multiexcitons. ${ }^{3,8,23,60}$ Signals observed by PL arise from the radiative relaxation of excited states. As such, the spectra are usually composed of a few identifiable transitions. Furthermore, the excited state lifetime is usually much longer than the time required for relaxation between excited states. In this common case, static PL only reveals the properties of the lowest emissive excited state. Time-resolved PL, however, allows the observation of higher lying emissive excited states, provided that the temporal resolution is faster than relaxation or that multiple independent radiative states are present. ${ }^{8,54,81}$

Previous experiments utilizing time-resolved PL up-conversion have extracted the emissive biexciton binding energy of CdSe quantum dots (QDs) through spectral shifts. ${ }^{8,82}$ Binding energies were in the range of $10-33 \mathrm{meV}$ for QDs of radii $1-3.5 \mathrm{~nm}$. It was found that initially, upon decreasing NC radius from $3.5 \mathrm{~nm}$ to $1.8 \mathrm{~nm}$, the binding energy increased from $14 \mathrm{meV}$ to $33 \mathrm{meV}$ before rapidly decreasing to $12 \mathrm{meV}$ upon further decreasing the radius. This trend inversion was attributed to the increased Coulombic repulsion between electron-electron and hole-hole pairs in the smaller NCs but has since been shown to be due to exchange interactions. $^{57}$ In addition to binding energies, multiexciton emission lifetimes were determined. These were found to be on the scale of 100-6 ps on decreasing QD radii, following the typical $r^{3}$ dependency found through transient-absorption spectroscopy. ${ }^{83}$ PL from single QD at high excitation intensity indicated the presence of up to quadraexcitons in CdTe/CdSe QDs. Multiple emission pathways are reported for TX, indicating a large structure in BX spanning $\sim 150 \mathrm{meV}^{56}$

Here, we use time-resolved PL (t-PL) to study the emission spectrum of excitons, biexcitons, and triexcitons. We perform t-PL using a streak camera, simultaneously resolving the emitted light in 
both energy and time. The t-PL spectrum depends on both the excitation energy $E_{\mathrm{ex}}$ and intensity $I_{0}$. The experimental t-PL signatures from CdSe NCs can be understood using the scheme in Fig. 4(a). At low excitation intensity, where $\langle N\rangle \ll 1$, a population of single excitons $\mathrm{X}$ is created. In this case, PL kinetics reveal the exciton lifetime $\tau_{\mathrm{X}} \sim 30 \mathrm{~ns}$. At high excitation intensity, two-photon absorption yields additional biexciton contributions with a lifetime $\tau_{\mathrm{BX}}$. The shift between PL from X and PL from BX reveals the emissive biexciton binding energy $\Delta_{\mathrm{Em}}\left(\mathrm{X}_{1} \mathrm{X}_{1}\right)$. At even higher intensities, multiphoton absorption allows the formation of multiexcitons (MX), as shown in Fig. 4(b), provided every exciton state encountered while climbing the ladder of states can absorb at $E_{\text {ex }}$. The MX can relax down the ladder of states, potentially yielding photons at every step, revealing the dynamics of the relaxation cascade.

a)

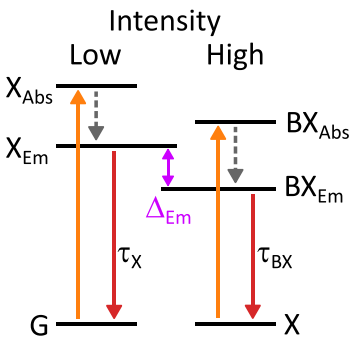

b)
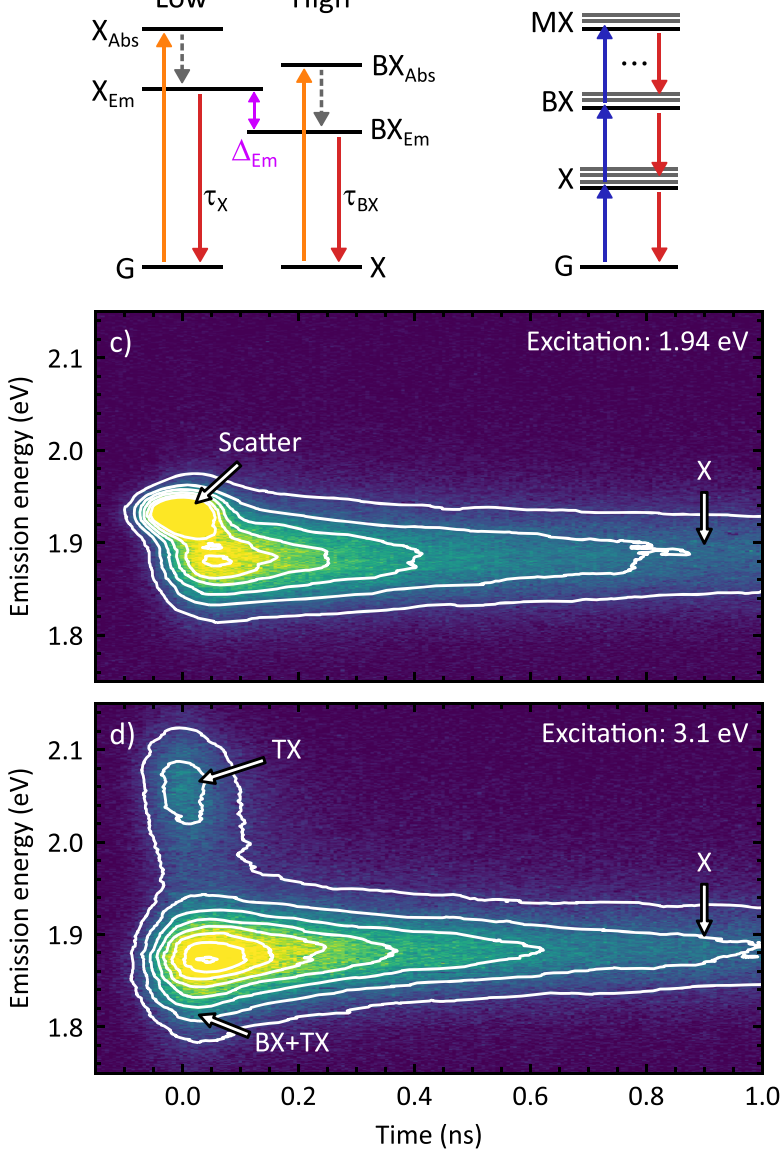

FIG. 4. Time-resolved photoluminescence spectra reveal the emission of relaxed excitons and biexcitons. (a) Level diagrams for exciton and biexciton. PL measures the effective binding energy between the lowest bright states of both manifolds. (b) Excitation using UV light allows the generation of multiple excitons. (c) Example $t-P L$ trace obtained using $X_{1}$ excitation. The signal is dominated by emission from $X$. Scattering from the excitation beam is also visible. (d) Example t-PL trace using high excitation intensity at $3.1 \mathrm{eV}$. The early part of the signal shows additional contributions, arising from bi- and tri-excitons.
Representative t-PL spectra with $\mathrm{X}_{1}$ excitation are shown in Fig. 4(c). The signal is dominated by emission from $\mathrm{X}_{1}$. Emission from $\mathrm{X}_{1} \mathrm{X}_{1}$ is present, but weak. The use of an excitation energy resonant with the $\mathrm{X}_{1}$ transition $(1.94 \mathrm{eV})$ prevents the formation of triexcitons. In CdSe QDs, the generation of more than two excitons cannot be achieved using band-edge excitation. The $1 S_{e}$ electron state has a degeneracy of two, and it is, thus, entirely filled in the $\mathrm{X}_{1} \mathrm{X}_{1}$ state. The next available electron state is $1 \mathrm{P}_{e}$, corresponding to the $\mathrm{X}_{3}$ transition. It requires significantly higher excitation energy. Scatter from the excitation beam is visible at $t=0 \mathrm{~ns}$. This contribution, which cannot be removed with simple filtering techniques, sets an upper technical limit on the excitation intensity: at high intensity, the scattered beam completely dominates the observed signal.

Additional features can be seen when using an excitation energy of $3.1 \mathrm{eV}$, shown in Fig. 4(d). The use of a high excitation energy enables the formation of multiexcitons as the NC exhibits strong absorption at this energy irrespective of its current exciton number. Similar to the previous case, the signal at longer times ( $>500 \mathrm{ps}$ ) is dominated by emission from single excitons, in agreement with Kasha's rule. However, additional contributions arising from TX are visible at early times on both the low and high energy sides as TX can relax via multiple pathways. These data are entirely consistent with previous reports obtained in similar conditions. ${ }^{59}$

A quantitative look at the fluence dependent kinetics can be achieved by integrating the t-PL spectrum as a function of emission energy. The resulting projections are shown in Fig. 5. Figure 5(a) shows the kinetic transients obtained by integrating t-PL traces for $1.77<E_{\mathrm{PL}}<1.895 \mathrm{eV}$, in order to eliminate scattering from the
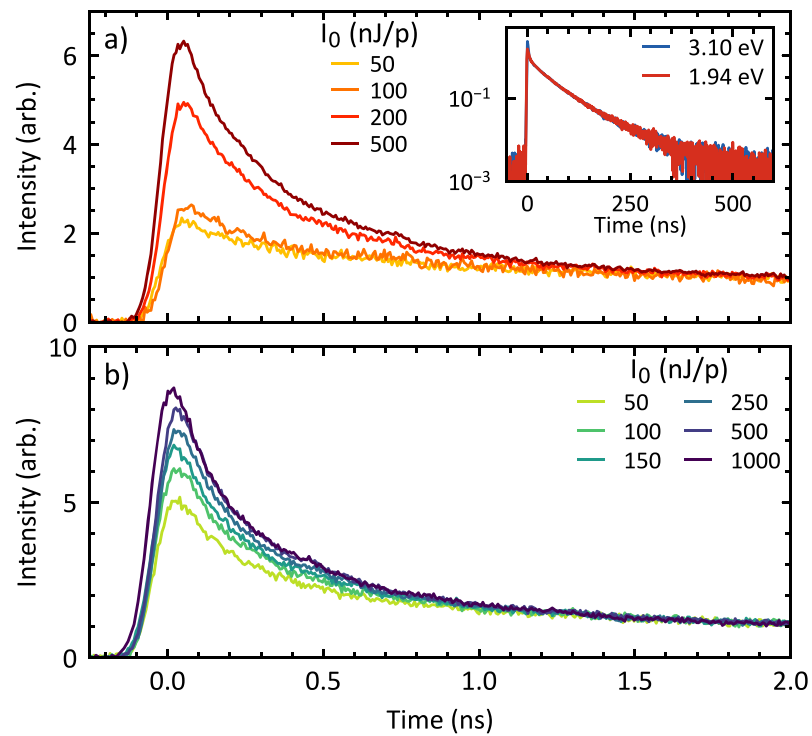

FIG. 5. Kinetic transients at increasing intensity reveal the emission from biexcitons. (a) Kinetic transients for $E_{\mathrm{PL}}<1.895 \mathrm{eV}$, using $1.94 \mathrm{eV}$ excitation. The biexciton component clearly increases at higher fluences. Inset: emission kinetics on longer time scales. (b) Kinetic transients for $E_{\mathrm{PL}}<1.895 \mathrm{eV}$ using a $3.1 \mathrm{eV}$ excitation. The early-time component increases with fluence, indicative of emission from $B X$ and $T X$. 
pump. The kinetic transients are normalized to their value at late time ( $t>5 \mathrm{~ns})$. We find that such a long time scale is necessary to observe the same dynamics irrespective of $I_{0}$. The relative intensity of the early time component increases monotonically with fluence, indicating they arise from BX. At low fluence, a weakly biexponential decay persists. We attribute this effect to multiple populations of NCs with different decay kinetics, the faster component corresponding to a dark fraction of NCs. ${ }^{84,85}$

The kinetic transients obtained with $3.1 \mathrm{eV}$ excitation are shown in Fig. 5(b), using the same procedure. Again, an early time component increases with fluence. Contrary to the previous case, the early time component does not vanish at low intensity, indicating multi-photon absorption is still present. ${ }^{86}$ This can be understood from the high absorption coefficient of NCs at $3.1 \mathrm{eV}$, and the high peak power of femtosecond pulses. Emission kinetics on longer time scales are shown in the inset of Fig. 5(a). The kinetics on this time scale are independent of intensity or excitation energy, indicating emission arises from the single exciton.

For $\mathrm{X}_{1}$ excitation, the biexciton signal can be obtained by comparing the signals obtained at low and high intensity excitation. This is achieved by scaling both images to their values at late time
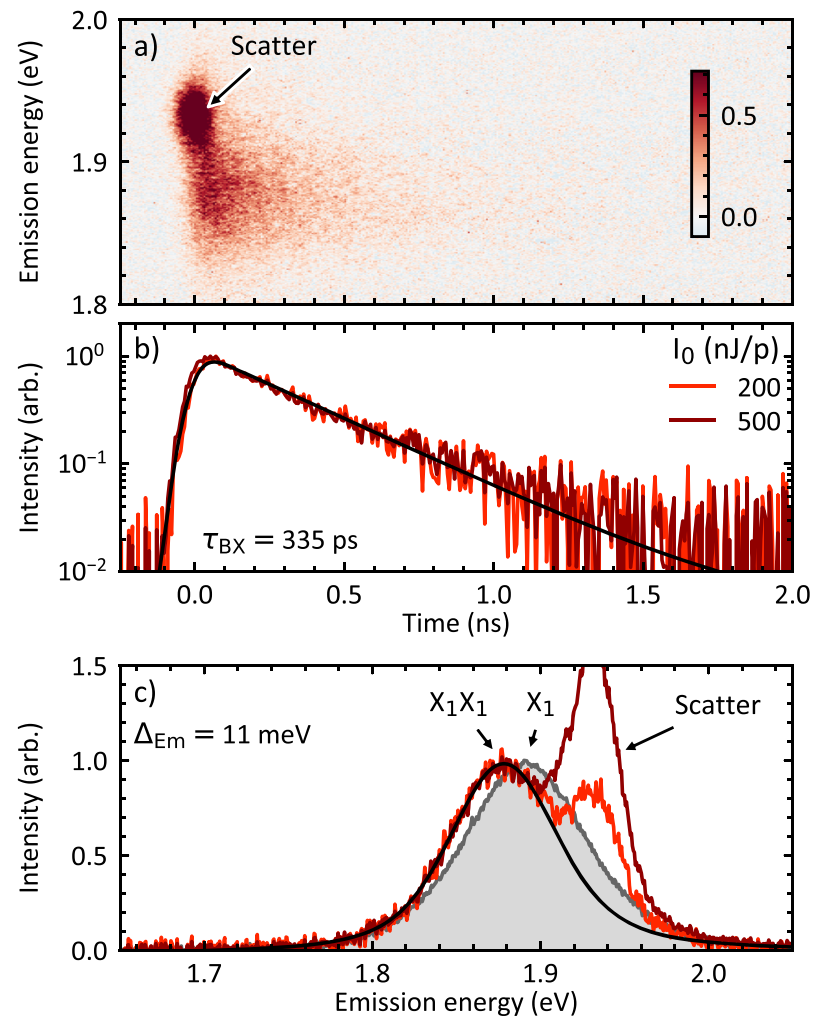

FIG. 6. Differential trace obtained by subtracting the low fluence signal from the high fluence signal using $1.94 \mathrm{eV}$ excitation. (a) The differential trace isolates the biexciton contribution. $I_{0}: 200 \mathrm{~nJ} / \mathrm{pulse}$. The color bar indicates values relative to streak trace before subtraction. (b) Biexciton kinetics are independent of $I_{0}$ with a lifetime of $335 \mathrm{ps}$. (c) Emission from the biexciton indicates a binding energy $\Delta_{\mathrm{Em}}\left(\mathrm{X}_{1} \mathrm{X}_{1}\right)=11 \mathrm{meV}$. The same legend is used to for panels (b) and (c). $(t>5 \mathrm{~ns})$ and performing a simple subtraction. The resulting differential streak trace is shown in Fig. 6(a). It shows two contributions: undesired scatter and emission signatures from $\mathrm{X}_{1} \mathrm{X}_{1}$. The kinetic transients are shown in Fig. 6(b). The resulting behavior is monoexponential, with a time constant of $\tau_{\mathrm{BX}}=335 \mathrm{ps}$, independent of intensity and emission energy. The black line shows a fit obtained by reconvolution fitting using a Gaussian IRF with FWHM 120 ps. The emission spectrum is shown in Fig. 6(c). The spectrum is entirely described by $\mathrm{X}_{1} \mathrm{X}_{1}$ emission and scatter from the excitation beam, both modeled as Gaussian peaks. The position of $\mathrm{X}_{1} \mathrm{X}_{1}$ emission does not depend on intensity. This behavior is consistent with the discrete nature of the exciton states and contrasts with the behavior of bulk semiconductors where the binding energy is sensitive to carrier density. The shift between the late-time spectrum (in gray) and the $\mathrm{X}_{1} \mathrm{X}_{1}$ emission yields $\Delta_{\mathrm{Em}}\left(\mathrm{X}_{1} \mathrm{X}_{1}\right)=11.5 \mathrm{meV}$. The single exponential temporal decay and simple emission spectrum, both independent of fluence, suggests that the simple scaling and subtraction approach neatly isolates the biexciton signature in this case.

The same procedure can be repeated for excitation using $3.1 \mathrm{eV}$ pulses. An example differential trace is shown in Fig. 7(a). In
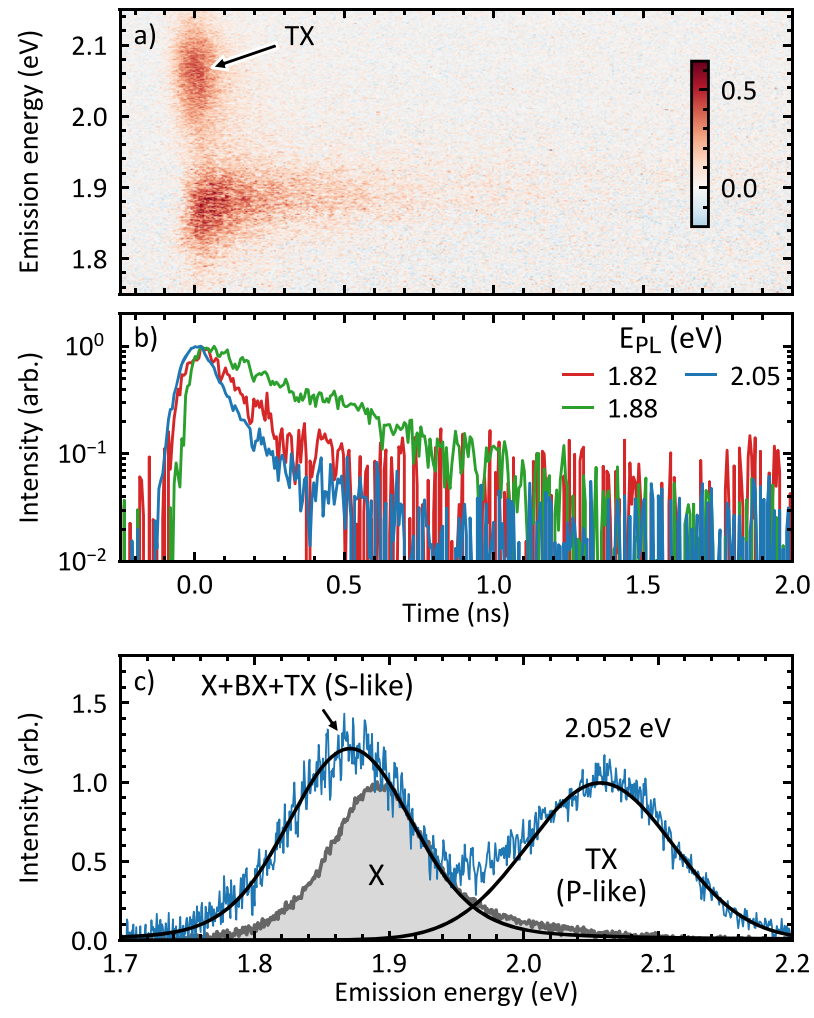

FIG. 7. The differential trace obtained using $3.1 \mathrm{eV}$ excitation reveals the triexciton (a) The differential trace contains signals arising from X, BX, and TX. $I_{0}: 500 \mathrm{~nJ} / \mathrm{p}$. The color bar indicates values relative to streak trace before subtraction. (b) The kinetics vary significantly across the trace. (c) The TX signal can be isolated, with an emission energy of $2.052 \mathrm{eV}$. The corresponding emissive triexciton binding energy is $139 \mathrm{meV}$ (see text). 
this case, however, the signal is more complicated and subtraction does not isolate a single contribution. Two general features can be observed: a short-lived signal between $2.0 \mathrm{eV}$ and $2.1 \mathrm{eV}$, labeled TX and a second feature below $1.9 \mathrm{eV}$, which blueshifts on a very short time scale. Relaxation kinetics vary as a function of $E_{\mathrm{PL}}$, as can be seen in Fig. 7(b). The duration of the TX feature is nearly IRF limited and uniform across the spectrum (blue curve). The kinetics in the feature below $1.9 \mathrm{eV}$ vary across the spectrum: the low energy edge is very short lived (red curve). The high energy end of this feature, around $1.9 \mathrm{eV}$, shows a significantly longer lifetime, which we attribute to a mixture of emission from BX and short-lived X population (green curve). This transient also shows a slight buildup delay. The short lived X component is visible at low fluence in Fig. 5(a) and has been discussed previously. The integrated spectrum obtained from the differential trace in panel (a) is shown in Fig. $7(\mathrm{c})$. The high energy TX feature has a central energy of $2.052 \mathrm{eV}$ irrespective of excitation intensity. The mixed feature at low energy is well modeled as a single Gaussian peak with a central position ranging from $1.86 \mathrm{eV}$ to $1.87 \mathrm{eV}$ with increasing excitation intensity. No attempts are made here to separate the contributions.

We assign the signals to a cascade of $\mathrm{TX} \rightarrow \mathrm{BX} \rightarrow \mathrm{X}^{59}$ The lowest energy TX that can be formed by optical excitation is $\mathrm{X}_{1} \mathrm{X}_{1} \mathrm{X}_{3}$ or $1 \mathrm{~S}_{e}^{2} 1 \mathrm{P}_{e} 1 \mathrm{~S}_{3 / 2}^{2} 1 \mathrm{P}_{3 / 2}$ in the electron-hole basis. The TX $\rightarrow$ BX process can occur via two radiative processes: recombination of an S-like exciton $\left(\mathrm{X}_{1} \mathrm{X}_{1} \mathrm{X}_{3} \rightarrow \mathrm{X}_{1} \mathrm{X}_{3}\right)$ or recombination of a P-like exciton $\left(\mathrm{X}_{1} \mathrm{X}_{1} \mathrm{X}_{3} \rightarrow \mathrm{X}_{1} \mathrm{X}_{1}\right){ }^{23,56}$ The S-like channel emits a photon of slightly lower energy than $\mathrm{X}_{1}$, consistent with the very fast kinetics at $E_{\mathrm{PL}}=1.82 \mathrm{eV}$. The second pathway yields photons with lower energy than $\mathrm{X}_{3}$, giving rise to the feature at $2.052 \mathrm{eV}$. This value represents a redshift of $128 \mathrm{meV}$ with respect to $\mathrm{X}_{3}$ absorption. We compute the emissive TX binding energy as the sum of this redshift and $\Delta_{\mathrm{Em}}\left(\mathrm{X}_{1} \mathrm{X}_{1}\right)$. The $\mathrm{BX}$ generated by recombination quickly relaxes to the lowest BX state, which is the same as for $1.94 \mathrm{eV}$ excitation. BX then recombines to $\mathrm{X}$. The mixed feature below $1.9 \mathrm{eV}$ is, thus, the addition of S-like recombination of TX, BX, and delayed X population. The two recombination pathways for TX differ in the final BX state. The energy for recombination by the S-like pathways cannot be determined accurately in these measurements. However, the two signals must be separated by $>170 \mathrm{meV}$. This is a clear indication of the coarse structure of BX.

Using t-PL, we could observe simultaneously signals arising from the radiative recombination of single excitons, biexcitons, and triexcitons. By varying both excitation energy and intensity, we could isolate signatures from all three processes. The emissive biexciton binding energy of $\mathrm{X}_{1}$ can be cleanly extracted upon resonant excitation and found to be $11 \mathrm{meV}$, in qualitative agreement with previous works. ${ }^{8,56}$ The lifetime of the biexciton is $\tau_{\mathrm{BX}}=335 \mathrm{ps}$. Short-lived signals arising from TX were observed with $E_{\mathrm{ex}}=3.1 \mathrm{eV}$. Defining the triexciton binding energy as the difference between the sum of the constituent single excitons and the triexciton, we can estimate a triexciton binding energy of $\Delta_{\mathrm{Em}}\left(\mathrm{X}_{1} \mathrm{X}_{1} \mathrm{X}_{3}\right)=139 \mathrm{meV}$. The analysis is carried out simply by scaling the t-PL traces to their late time intensity, followed by subtraction.

Time-resolved PL is uniquely powerful to observe multiexcitons as it allows us to study the excited states in the absence of a background arising from the ground state. The simultaneous spectral and temporal resolution enables the isolation of signals from multiple MX and the observation of dynamics on time scales ranging from 100 ps to 100 ns. However, observations made by PL are limited to the spontaneously emissive states, which are generally the lowest energy state of a given manifold. In order to address in detail the electronic structure of multiexcitons in nanostructures, complementary information obtained using other techniques is needed.

\section{State-resolved pump-probe}

The use of experiments based on PL enabled the evaluation of the properties of the lowest emissive exciton and multiexciton states. In order to gain information on higher lying biexcitons, methods which probe the absorptive spectroscopic response are necessary. Optical pump-probe spectroscopy is a well-established femtosecond spectroscopy technique which enables the interrogation of excited states. By adjusting the properties of the excitation beam, the initial state can be specified. We call this technique state-resolved pump-probe (SRPP).

Pump-probe spectroscopy is one of the most common tools used to study ultrafast dynamics in the excited states of optically active materials. A short pump pulse is used to excite the system, and after a certain controlled delay, a second pulse probes the excited system. By varying the delay between pump and probe pulses, the relaxation of the excited state toward equilibrium can be followed. Importantly, the temporal resolution in a pump-probe experiment is limited by the duration of the pump and probe pulses not by the detector response time. This allows for experiments with femtosecond resolution that use slow photodiode or CCD based detectors.

We perform an optical-pump, optical-probe experiment in transmission mode. In this experiment, the transmission of the broadband probe pulse is monitored as a function of pump-probe delay. It is, thus, transient absorption of the sample that is probed. A probe pulse will have different transmission if the sample was excited by a pump pulse or not. This is transient absorption (TA) spectroscopy. The TA spectrum has three components, shown in Fig. 8(a):

1. Ground state bleach (GSB). This is due to depletion of the ground state. GSB will decrease absorption, i.e., a negative signal in the $\triangle \mathrm{OD}$ spectrum. The intensity of this signal decreases as the ground state population recovers.

2. Stimulated emission (SE). This is due to stimulated emission through radiative recombination of a bright excited state. SE is another negative feature in the $\triangle \mathrm{OD}$ spectrum. This signal reveals dynamics in the excited state.

3. Excited state absorption (ESA). This is due to absorption from one excited state to a higher lying state such as biexcitons. This causes an increase in absorption, a positive feature in a $\Delta \mathrm{OD}$ spectrum. This signal also tracks the dynamics in the excited state manifold.

An example dataset is shown in Fig. 8(b). A cut at fixed delay yields a transient spectrum, as shown in Fig. 4(c). TA signals depend on the properties of the exciting beam. State-resolved pump-probe (SRPP) exploits this behavior to prepare different excited states. By pumping into different initial states, qualitatively different effects can be observed, including gain, ${ }^{26,87-89}$ carrier cooling, ${ }^{90,91}$ and non-radiative trapping channels. ${ }^{53,92-95}$ Furthermore, by carefully 
a)
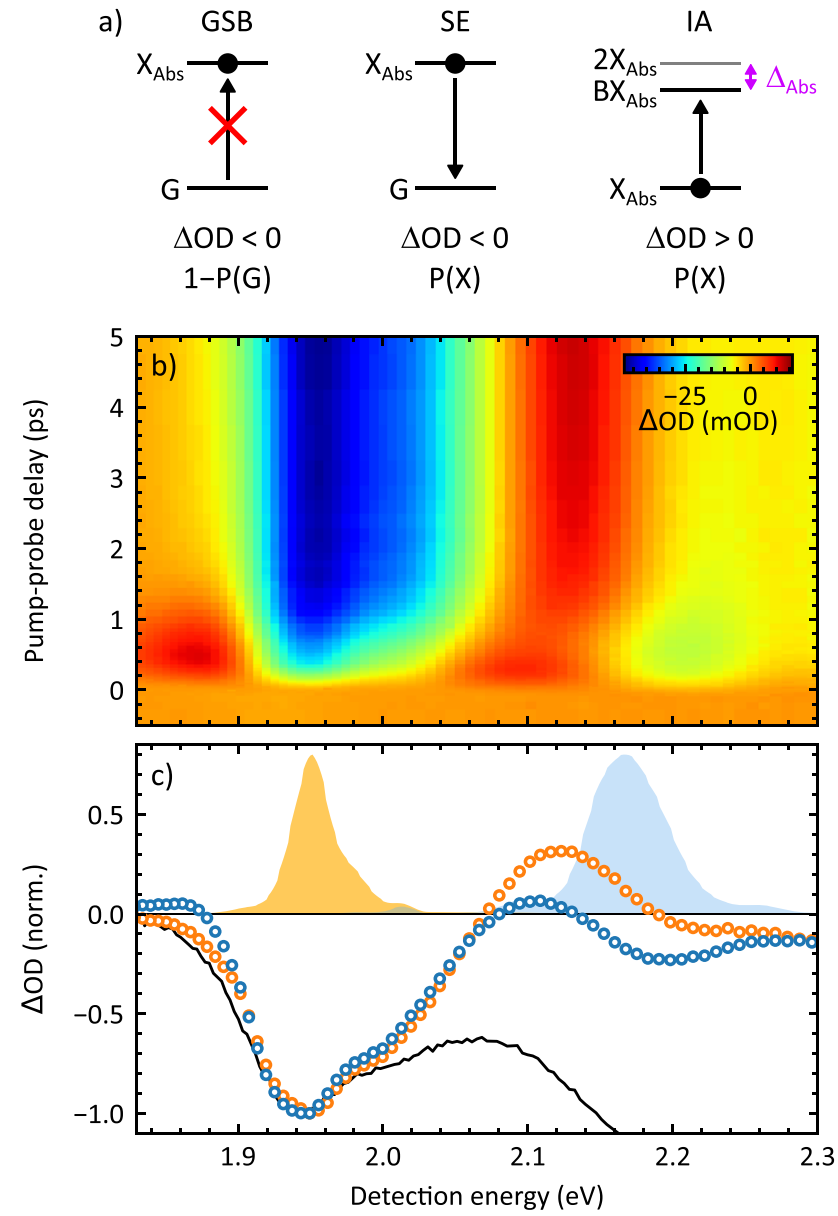

FIG. 8. SRPP measure changes in the absorption spectrum. (a) Contributions to the signal: ground-state bleach (GSB), stimulated emission (SE), and excited state absorption (ESA). (b) Example TA dataset obtained using $3.1 \mathrm{eV}$ excitation. It reports the change in absorption $(\triangle O D)$ vs detection energy and pumpprobe delay. (c) The excitation energy controls the initial state of the system, thus enabling state-resolved pump-probe. Signals for $X_{1}$ excitation and $X_{3}$ excitation are shown. The negative of the linear signal absorption signal is shown for reference (black line).

choosing the initial state, hole-specific or electron-specific effects can be observed. ${ }^{90}$ These considerations make SRPP a powerful tool to study quantum confined systems. Our group has developed an instrument that performs two pump-probe experiments in parallel, ${ }^{71}$ allowing quantitative comparison of the dynamics induced by two different excitation energies. Figure 4(c) shows two transient spectra obtained with two different excitation conditions: $\mathrm{X}_{1}$ (orange) and $\mathrm{X}_{3}$ (blue).

The TA spectrum arises from a number of overlapping contributions, which must be separated to extract biexciton binding energies. The TA lineshapes result from an interplay of the linewidths and binding energies. This is illustrated in Fig. 9. The net result can range from a slight peak shift $\left(\mathrm{X}_{1}\right)$ to a derivative lineshape $\left(\mathrm{X}_{3}\right) .{ }^{10,80}$ For the band-edge exciton, there are two negative signals

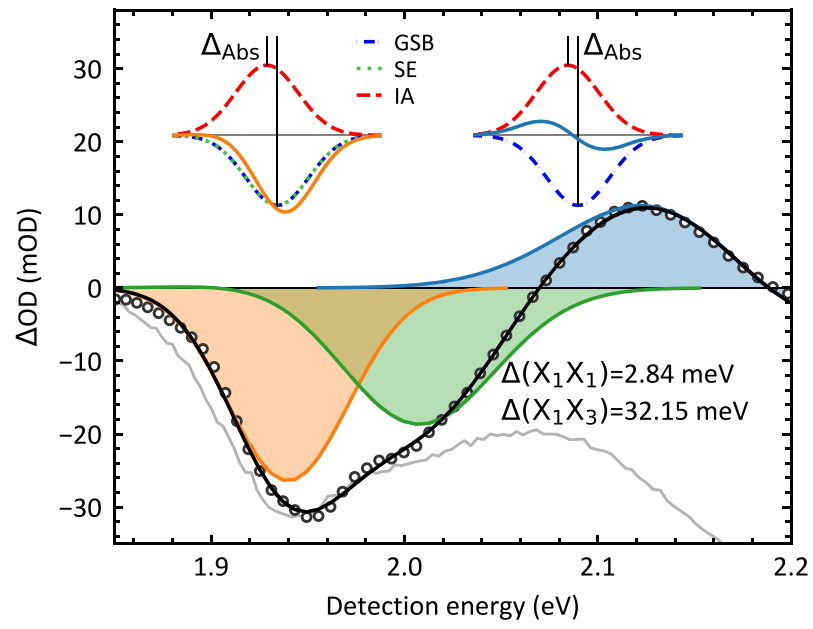

FIG. 9. Analysis of the transient absorption spectrum at low intensity. Depending on the transition, biexcitons result in either attenuated bleach (left) or a derivative lineshape (right). Analysis of the TA spectrum enables the extraction of binding energies for $X_{1} X_{i}$ biexcitons. Spectrum obtained with $X_{1}$ excitation, a delay of 5 ps and $I_{0}=90 \mathrm{~nJ} /$ pulse.

in the same position (GSB, SE) and a single positive signal with a slight redshift (ESA). All contributions are of similar magnitude. The net result is a slight blueshift of the resultant peak with respect to the linear absorption spectrum, known as attenuated bleach. This attenuated bleach is visible in Figs. 8(c) and 9. The situation is different for the $\mathrm{X}_{3}$ transition. The signal arises from two contributions of comparable magnitude: GSB and ESA. The result is a derivative lineshape.

The biexciton binding energy can be extracted from the TA spectrum as shown in Fig. 9 for low intensity, $\mathrm{X}_{1}$ excitation with a pump-probe delay of 5 ps. The TA spectrum arises from NCs in $X_{1}$. The contributions are, thus, GSB, SE from $\mathrm{X}_{1}$, and ESA from $\mathrm{X}_{1}$ to $\mathrm{X}_{1} \mathrm{X}_{i}$ biexcitons. Biexciton binding energies are extracted from this spectrum using a fitting procedure previously developed by Zhang. ${ }^{80}$ The model in Eq. (3) is fitted to the transient absorption spectrum, $\Delta \mathrm{OD}(E)$,

$$
\begin{gathered}
\Delta \mathrm{OD}(E)=A_{0}[-\mathrm{GSB}(E)-\mathrm{SE}(E)+\mathrm{ESA}(E)], \\
\mathrm{GSB}(E)=\sum_{i=1}^{3} \alpha_{i} G\left(E, E\left(\mathrm{X}_{i}\right), \sigma_{i}\right) \\
\mathrm{SE}(E)=\alpha_{1} G\left(E, E\left(\mathrm{X}_{1}\right), \sigma_{1}\right) \\
\operatorname{ESA}(E)=\alpha_{1} m_{1} G\left(E, E\left(\mathrm{X}_{1}\right)-\Delta_{1}, \sigma_{1}\right) \\
+\frac{1}{2} \alpha_{2} m_{2} G\left(E, E\left(\mathrm{X}_{2}\right)-\Delta_{2}, \sigma_{2}\right) \\
+\alpha_{3} m_{3} G\left(E, E\left(\mathrm{X}_{3}\right)-\Delta_{3}, \sigma_{3}\right)
\end{gathered}
$$

$A_{0}$ is an arbitrary scaling factor. $\operatorname{GSB}(E)$ is equal to the modeled linear absorption spectrum using Eq. (1) shown in Fig. 3, without the polynomial background. Stimulated emission is assumed to only occur from the $\mathrm{X}_{1}$ state; therefore, $\mathrm{SE}(E)$ is defined by a single Gaussian with the same amplitude, width, and position as the linear absorption peak corresponding to $\mathrm{X}_{1}$. While fitting the 
model, the $\operatorname{GSB}(E)$ and $\operatorname{SE}(E)$ terms are uniquely defined by the linear absorption spectrum and not varied. The ESA is modeled as a series of three Gaussian peaks with the same widths as in $\mathrm{GSB}(E)$ although their amplitudes are scaled, respectively, by factors $m_{i}=\left|\mu_{\mathrm{X}_{1} \rightarrow \mathrm{X}_{1} \mathrm{X}_{i}}\right|^{2} /\left|\mu_{G \rightarrow \mathrm{X}_{1}}\right|^{2}$. Likewise, the positions of the ESA peaks are shifted, respectively, from the $\mathrm{X}_{1}, \mathrm{X}_{2}$, and $\mathrm{X}_{3}$ states by biexciton binding energies $\Delta_{i}=\Delta_{\mathrm{Abs}}\left(\mathrm{X}_{1} \mathrm{X}_{i}\right)$. The amplitude of ESA from $\mathrm{X}_{1}$ to $\mathrm{X}_{1} \mathrm{X}_{2}$ is divided by two to account for the occupied $1 \mathrm{~S}_{e}$ electron [Eq. (6)]. This effect, obvious in the electron-hole representation, is known as Pauli blocking in the exciton basis. ${ }^{29,80}$ The results of this fitting procedure are shown in Fig. 9. We note that the ESA signal from $\mathrm{X}_{1} \mathrm{X}_{2}$ is not clearly visible, unlike the clear signatures of ESA from $\mathrm{X}_{1} \mathrm{X}_{1}$ (attenuated bleach) and $\mathrm{X}_{1} \mathrm{X}_{3}(\Delta \mathrm{OD}>0)$. As such, the value of $\Delta_{2}$ is not statistically robust. The other binding energies are $\Delta_{\mathrm{Abs}}\left(\mathrm{X}_{1} \mathrm{X}_{1}\right)=2.84 \mathrm{meV}$ and $\Delta_{\mathrm{Abs}}\left(\mathrm{X}_{1} \mathrm{X}_{3}\right)=32.15 \mathrm{meV}$, in agreement with previous results. ${ }^{80}$ This method successfully models the TA spectrum and allows a measurement of the binding energy for a series of mixed excitons. This is achieved by a careful evaluation of the contributions and by determining the parameters in two steps: exciton properties are determined from the linear spectrum; then, biexciton properties are determined from the TA spectrum.

The signatures of biexcitons can also be observed by increasing excitation intensity $I_{0}$ in order to directly generate a population in the biexciton state. The band-edge spectrum as a function of intensity is shown in Fig. 10(a). The position of the peak shifts to lower energies with increasing intensity. The use of the
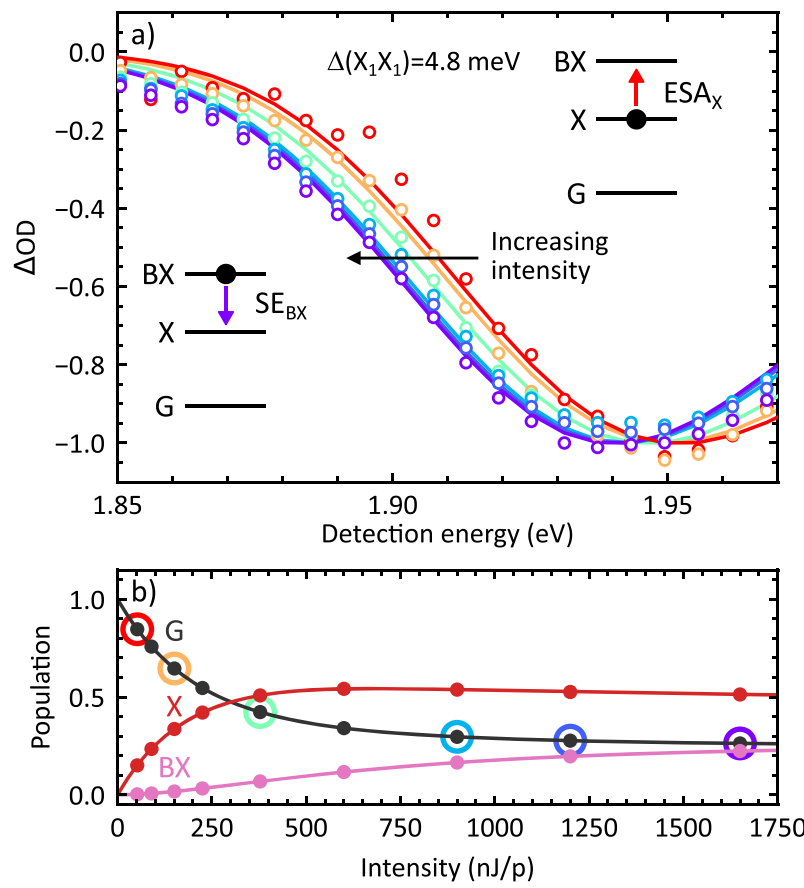

FIG. 10. Extraction of an intensity independent binding energy. (a) At high fluences, a significant population is present in BX, resulting in an intensity dependant redshift. Solid lines are fit to a global model (see text). (b) Model intensity dependent populations in the ground state $(G)$, exciton $(X)$, and biexciton (BX) manifolds. previous spectral model would yield a binding energy that depends on $I_{0}$, inconsistent with results from t-PL and with theoretical models. An intensity independent binding energy can be obtained by modeling the intensity dependant band-edge spectrum. The data and results are shown in Fig. 10(a). For low fluence, when $\langle N\rangle \ll 1$, the signal arises only from GSB, SE $\left(\mathrm{X}_{1} \rightarrow G\right)$, and ESA $\left(\mathrm{X}_{1} \rightarrow \mathrm{X}_{1} \mathrm{X}_{1}\right)$. At high fluence, however, a fraction of the NCs will undergo twophoton absorption to form biexcitons. For the biexciton population, the ESA signal is converted to $\mathrm{SE}\left(\mathrm{X}_{1} \mathrm{X}_{1} \rightarrow \mathrm{X}_{1}\right)$ and, thus, flips sign. As a result, the peak of the TA signal shifts toward lower energies with increasing $I_{0}$. This variation of the spectral shape with fluence adds additional constraints during the fitting procedure. It is, thus, possible to optimize the linewidth $\sigma_{1}$ to account for size distribution effects, independently of the linear absorption spectrum.

The transient absorption spectra were obtained with the use of an excitation beam resonant with the $\mathrm{X}_{1}$ transition. This situation is different from the more common case of excitation using $3.1 \mathrm{eV}$, in the continuum. For continuum excitation, the absorption cross section of an individual $\mathrm{NC}$ is independent of its electronic state. The distribution of the number of excitons per particle, thus, fol-

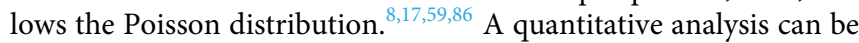
made, including corrections for experimental geometry. ${ }^{86}$ However, the use of $3.1 \mathrm{eV}$ excitation generates hot excitons which can then follow a number of different paths. This gives rise to a mixture of NC states. In order to reduce this effect and better specify the excited state populations, we choose to excite resonantly with $\mathrm{X}_{1}$.

The series of fluence dependent spectra in Fig. 10(a) are globally fitted using a model comprising an intensity-dependent population part and a spectral part,

$$
\Delta \mathrm{OD}\left(E, I_{0}\right)=\left[1-n_{0}\left(I_{0}\right)\right] S_{0}(E)+n_{1}\left(I_{0}\right) S_{1}(E)+n_{2}\left(I_{0}\right) S_{2}(E),
$$

where $n_{i}$ is the population fraction in state $i$ after excitation $\left(n_{0}=G\right.$, $\left.n_{1}=\mathrm{X}, n_{2}=\mathrm{BX}\right), S_{i}(E)$ is the spectrum of state $I$, and $I_{0}$ is the excitation intensity. The spectra are

$$
\begin{gathered}
S_{0}(E)=-\mathrm{GSB}(E), \\
S_{1}(E)=-\mathrm{SE}_{\mathrm{X}}(E)+\operatorname{ESA}_{X}(E), \\
S_{2}(E)=-\operatorname{SE}_{\mathrm{BX}}(E)=\alpha_{1} m_{1} G\left(E, E\left(\mathrm{X}_{1}\right)-\Delta_{1}, \sigma_{1}\right) .
\end{gathered}
$$

$\mathrm{GSB}(E)$ is the ground state spectrum [Eq. (4)], $\mathrm{SE}_{\mathrm{X}}(E)$ is $\mathrm{X}_{1} \rightarrow \mathrm{G}$ stimulated emission [Eq. (5)], $\mathrm{ESA}(E)$ is $\mathrm{X}_{1} \rightarrow \mathrm{X}_{1} \mathrm{X}_{1}$ absorption [Eq. (6)], and $\mathrm{SE}_{\mathrm{BX}}(E)$ is $\mathrm{X}_{1} \mathrm{X}_{1} \rightarrow \mathrm{X}_{1}$ stimulated emission. This spectral model contains the exact same parameters as the model used for low fluence.

The population fractions are obtained using a saturation model for a 3-level system under the action of a Gaussian pulse, including both absorption and stimulated emission,

$$
\begin{gathered}
n_{i}\left(I_{0}\right)=n_{i}(t \rightarrow \infty), \\
g_{i} \dot{n}_{i}(t)=a I_{0} \exp \left[-0.5\left(t / \sigma_{t}\right)^{2}\right] \sum_{j=0}^{2} k_{i j} n_{j}(t),
\end{gathered}
$$

where $g_{i}$ is the degeneracy of state $i, t$ is the time, $a$ is a proportionality constant, and $I_{0}$ is the excitation intensity. We use a doubly degenerate exciton $\left( \pm 1^{L}\right.$ state $) .^{61}$ The rate matrix contains the 
effective light-induced transition rates,

$$
\boldsymbol{k}=\left[\begin{array}{ccc}
-k_{1} & k_{1} & 0 \\
k_{1} & -k_{1}-k_{2} & k_{2} \\
0 & k_{2} & -k_{2}
\end{array}\right],
$$

where $k_{1}$ is the transition rate for the $\mathrm{G} \leftrightarrow \mathrm{X}$ transition and $k_{2}=r k_{1}$ is the transition rate for the $\mathrm{X} \leftrightarrow \mathrm{BX}$ transition. This equation of motion is integrated numerically using $\boldsymbol{n}(0)=[1,0,0]$ as initial conditions to yield the populations in Eq. (7). We set $\sigma_{t}=100 \mathrm{fs}$ and $k_{1}=1$. This has no impact on the results as the change is absorbed by the proportionality constant $a$. The saturation model, thus, has two adjustable parameters: the proportionality constant $a$ and the ratio $r$.

The global model is fitted to the low energy edge of the transient spectra. A total of nine intensities are used, without normalization. The residuals from each spectrum are weighted by the inverse of their peak value in order to capture the spectral shift. The absorption cross sections $\alpha_{i}$ are not varied from their values determined using the linear absorption spectrum. The following parameters do not impact the values in the window of interest and are kept fixed: $E_{2}, \sigma_{2}, E_{3}, \sigma_{3}$, and $\Delta_{3}$.

The global model has a single extra parameter compared to the model described in Eqs. (3)-(6), yet it captures the intensity dependent shift of the band-edge peak, as shown in Fig. 10(a). The fitted population dynamics are shown in Fig. 10(b). The resulting binding energy is $\Delta_{\mathrm{Abs}}\left(\mathrm{X}_{1} \mathrm{X}_{1}\right)=4.84 \mathrm{meV}$. This model explicitly neglects relaxation and trapping, which occur on time scales longer than studied here.

State-resolved pump-probe is a powerful technique to reveal the structure of multiexcitons. At low excitation intensity, biexcitons can be observed via excited state absorption into a manifold of biexciton states. The clearest experimental signatures are positive values of $\triangle \mathrm{OD}$ and attenuated bleach on the low energy edge of the spectrum. At high excitation density, stimulated emission from a population of biexcitons can be observed via an intensity dependent shift of the first peak. Due to the large number of overlapping contributions, a number of approximations and assumptions have to be made with regard to the fine-structure, relaxation dynamics, and line broadening mechanisms.

\section{Two-dimensional spectroscopy}

So far, we have presented how t-PL measurements yields direct information about the emissive states of biexcitons and multiexcitons. We have also shown that state-resolved TA spectroscopy yields complementary information about the absorptive structure of biexcitons. Binding energies were extracted from fitting TA spectra, yet several assumptions were made in the process. Given the small values of the binding energies, we observed that a slight variation of the fit parameters could yield significant changes in the extracted values. More generally, due to the size dispersion of CdSe NCs, an important limitation of TA spectroscopy is its inability to clearly disentangle biexciton effects from size heterogeneity effects. By virtue of spreading the optical response along two-dimensions, the 2DE method is, in principle, capable of overcoming this challenge. In this section, we explore the strengths and weaknesses of $2 \mathrm{DE}$ spectroscopy with regard to probing the absorptive structure of biexcitons.
The 2DE experiments conducted here are analogous to a pump-probe experiment where the pump is passed through an interferometer before interacting with the sample. It has been presented in detail previously. ${ }^{96-99}$ The interferometric pump pair enables the resolution of the excitation energy within the pulse bandwidth by Fourier transform spectroscopy. The experiment uses a series of three pulses, schematically represented in Fig. 11(a). The first two pulses are separated by delay $t_{1}$. The second and third pulses are separated by delay $t_{2}$. The experiments are performed in the pump-probe geometry: the first two pulses are collinear, on the same beam path. The pumps and probe cross in the sample, as in regular TA experiments. ${ }^{33,72,100,101}$ The transmitted spectrum from pulse three is monitored as a function of the delay $t_{1}$ and $t_{2}$. A spectrometer records the intensity at wavelength $\lambda_{3}$. The $2 \mathrm{D}$ signal depends on the relative phases of the first two pulses $\Delta \varphi_{12}$, whereas the linear and pump-probe signals do not. This enables the isolation of the $2 \mathrm{D}$

a)

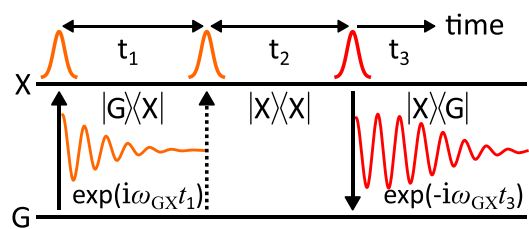

b)
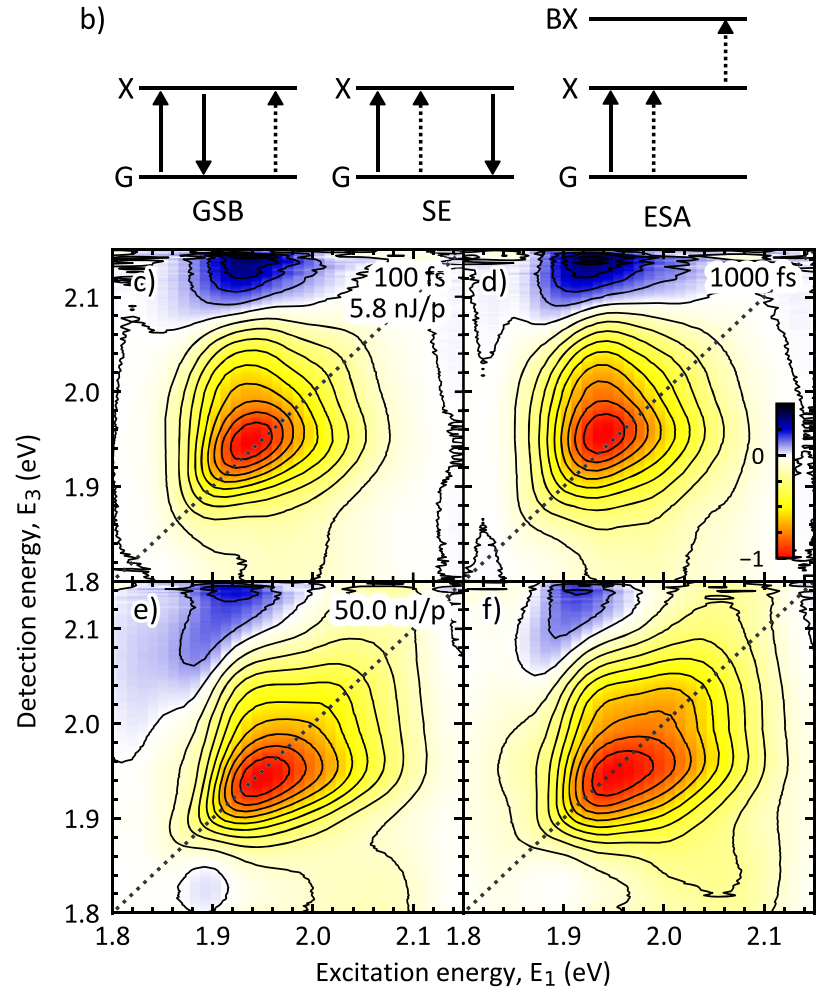

FIG. 11. Two dimensional spectroscopy resolves the electronic response along excitation energy $E_{1}$, delay $t_{2}$, and detection energy $E_{3}$. (a) $2 D E$ pulse sequence. Solid (dashed) arrows indicate transitions of the bra (ket). (b) Diagrams for GSB, $\mathrm{SE}$, and ESA processes. (c)-(f) Overview of the 2D spectra as a function of pump energy and delay (see legend). The 2D spectra show a significant distortion at high energy, arising from two-photon absorption. 
signal by phase cycling. ${ }^{74,96,102}$ The signal recorded at multiple values of $\Delta \varphi_{12}$ is linearly combined to isolate the $2 \mathrm{D}$ signal. The resulting dataset has three axes: $t_{1}, t_{2}$, and $\lambda_{3}$. The $2 \mathrm{D}$ spectra are obtained by Fourier transformation along $t_{1}$ to yield an excitation axis. The detection energy $E_{3}$ is obtained directly from $\lambda_{3}$.

$2 \mathrm{D}$ spectroscopy enables resolution along the excitation axis by separating the excitation process in two steps. The experimental scheme is directly analogous to the calculation of the third order response. ${ }^{62}$ The first pulse creates a coherent superposition of quantum states between the ground state $\mathrm{G}$ and excitonic states $\mathrm{X}_{i}$ (e.g., $\left.|G\rangle\left\langle X_{1}\right|\right)$. This coherence evolves during time period $t_{1}$. This coherent evolution is sensitive to the relative phase of the pump pulses. The second pulse converts the coherence into a population which evolves during time period $t_{2}$, sometimes called population time. A last pulse is used to probe the sample, creating again a coherence which evolves during time period $t_{3}$. This last coherence generates a macroscopic polarization of the sample which radiates the signal field. In the pump-probe geometry, the signal field is emitted in the same direction as the probe beam. A $2 \mathrm{DE}$ spectrum is obtained at a given population time $t_{2}$ by performing Fourier transformations along the $t_{1}$ and $t_{3}$ axes. Delay $t_{1}$ yields the excitation energy $E_{1}$, and delay $t_{3}$ yields the detection energy $E_{3}$. In the pump-probe geometry, the Fourier transform along $t_{3}$ is performed by the spectrometer. More details about the 2DE method can be found in reference texts. $^{62,96-98,103}$

The physical processes contributing in a $2 \mathrm{DE}$ spectrum are identical to the contributions in a TA experiment. The contributions are illustrated in Fig. 11(b). The three different signals arise from a different series of interactions between the sample and the light pulse. The possible sequences of light-matter interactions can be represented as double-sided Feynman diagrams (DSFD) or as wave-mixing energy level (MWEL) diagrams. We choose the latter here as they are directly related to the common Jablonsky diagrams. The horizontal bars represent the eigenstates of the material Hamiltonian. Time runs from left to right. Arrows indicate lightmatter interactions. Solid arrows represent interactions of light with the ket side of the density matrix; dashed arrows represent interactions with the bra. On the first diagram, the density matrix, thus, goes though the sequence $|G\rangle\langle X|\rightarrow| G\rangle\langle G|\rightarrow| X\rangle\langle G|$. This contribution tracks the dynamics occurring in the ground state during delay $t_{2}$, and it is the GSB signal. The other two diagrams project the state into an excited state population during delay $t_{2}$. They differ in their interaction with the third pulse. In SE, it stimulates relaxation toward the ground state, and in ESA, it induces mixing with doubly excited states. These contributions to the $2 \mathrm{D}$ signal are identical to the signals contributing to a TA spectrum. Indeed, a TA measurement can be obtained from a $2 \mathrm{D}$ experiment by setting $t_{1}=0$ or equivalently by integrating over $E_{1}$. Similarly, a slice at fixed $E_{1}$ is called a pseudo-TA spectrum. The $2 \mathrm{DE}$ spectra can, thus, be interpreted just like in a TA experiment in terms of GSB, SE, and ESA.

Information about the biexciton is obtained from ESA signals. While TA and 2DE are sensitive to the same physical processes, there are a number of important differences in the observables of both methods. A key difference is that the pump energy resolution in a TA experiment is given by the spectral width of the OPA pulse. In contrast, the pump energy resolution in a $2 \mathrm{DE}$ experiment ( $E_{1}$ axis) is dictated by the time range of the coherence time delay $t_{1}$. This yields a typical energy resolution along the $E_{1}$ axis of $10 \mathrm{meV}$. In a $2 \mathrm{DE}$ experiment, there is no inherent trade-off between energy and time resolution. This enables to access population dynamics with $\simeq 10$ fs resolution, while maintaining an excellent energy resolution along $E_{1}$. Another important difference is that the inhomogeneous and homogeneous contributions to the lineshape are clearly separated on a $2 \mathrm{D}$ spectrum, unlike in one-dimensional TA spectra. Static inhomogeneous broadening, for example, arising from size distribution, stretches the signal along the diagonal of the spectrum. ${ }^{104,105}$ All these methodological advantages are highly relevant for the observation of biexcitonic effects in ensembles of CdSe QDs.

Exemplary 2DE spectra of CdSe QDs are shown in Figs. 11(c)-11(f) for two representative population times $t_{2}$ and excitation intensity $I_{0}$. We first describe the signal in panel (c). The central feature on the diagonal arises from the $\mathrm{X}_{1}(1.94 \mathrm{eV})$ and $\mathrm{X}_{2}$ $(2.00 \mathrm{eV})$ transitions. These two transitions are strongly coupled due to their shared electron state. ${ }^{29}$ As such, they give rise to four overlapping signals: two diagonal signals and two cross peaks, located at the coordinates of the $\mathrm{X}_{1}$ and $\mathrm{X}_{2}$ transitions. All contributions are negative as they arise from GSB and SE. The signals involving $\mathrm{X}_{1}$ are stronger, consistent with both linear spectra [Fig. 3(c)] and SRPP with $\mathrm{X}_{1}$ excitation [Figs. 8(c) and 9]. Each of the four features is elongated diagonally by inhomogeneous broadening. The resulting peak has a blunt arrowhead shape pointing to the lower left. A positive ESA is visible at $E_{3}>2.08 \mathrm{eV}$, consistent with ESA to $\mathrm{X}_{1} \mathrm{X}_{3}$ in TA. The zero-crossing line, separating the domains dominated by bleach and ESA, is almost horizontal. These results are fully consistent with decades of TA spectroscopy and with previous 2DE investigations on similar materials. ${ }^{24,29,30,32,33}$

Increasing $I_{0}$ gives rise to a number of changes of the $2 \mathrm{DE}$ spectra. The excitation is performed by two pulses, and the results from $2 \mathrm{DE}$ are, thus, equivalent to a SRPP experiment performed with twice the intensity. The ESA contributions (blue) change with increasing fluence. In particular, at $100 \mathrm{fs}$, the high-fluence spectrum shows a positive signal to the red of the band-edge peak position $\left(E_{1}=1.89 \mathrm{eV}, E_{3}=1.82 \mathrm{eV}\right)$. Furthermore, the ESA feature at $E_{3}=2.12 \mathrm{eV}$ changes shape: whereas it is mostly horizontal at low intensity, it acquires a strongly diagonal character at high excitation intensity. In addition to the changes in ESA signatures, one can also observe changes in the band-edge peak shape. The central feature stretches horizontally toward higher values of $E_{1}$. All these features contain information about the biexciton absorptive structure, which we describe in detail below.

In order to extract biexcitonic contributions in the presence of the strong static inhomogeneity arising from size dispersion, a key aspect is to exploit the increased energy resolution along $E_{1}$. This can be illustrated by taking pseudo-TA projections. These projections are obtained by selecting a value of $E_{1}$ and reporting the $2 \mathrm{D}$ signal as a function of $E_{3}$ at a fixed value of $t_{2}$. Figure 12(a) illustrates this point by representing exemplary pseudo-TA projections for increasing values of $E_{1}$. On this plot, one observes that the bandedge peak position blueshifts as $E_{1}$ increases, reflecting the different sub-populations of NCs within the ensemble. By analyzing the redmost part of the $2 \mathrm{D}$ spectrum, one can, thus, access specific signals which would otherwise be obfuscated.

Figures 12(b) and 12(c) show zoomed-in 2D spectra to the red of the band-edge peak for the case of $10 \mathrm{~nJ} /$ pulse excitation. The 

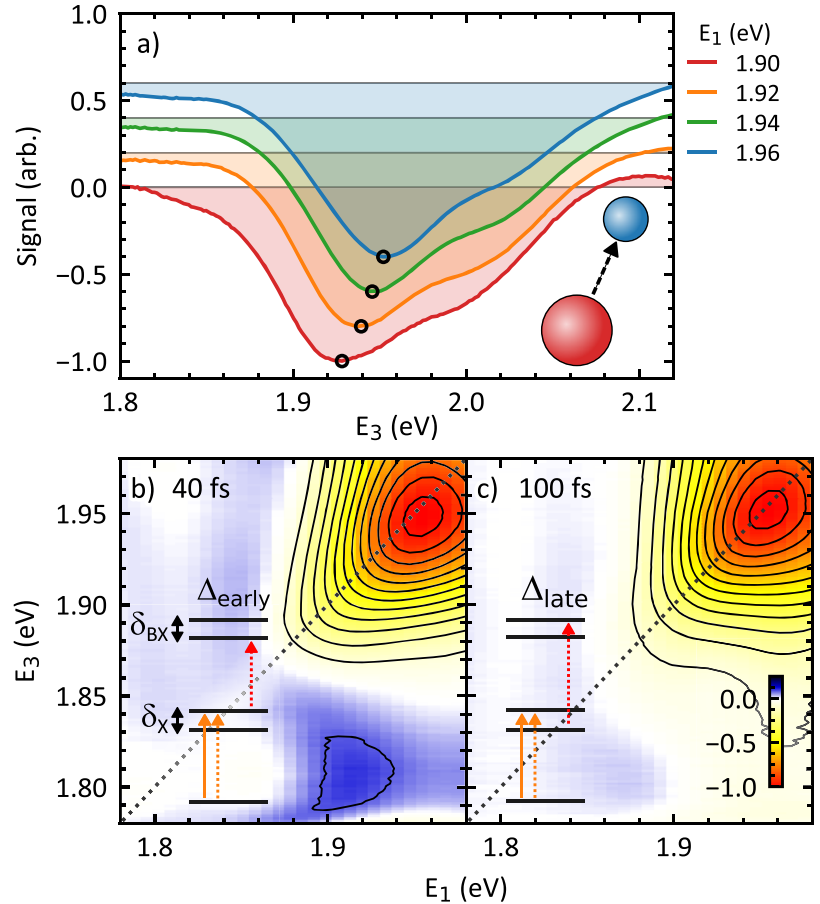

FIG. 12. 2DE resolves biexction absorption in the presence of inhomogeneous broadening. (a) Pseudo-TA slices taken at increasing excitation energy reveals inhomogeneous broadening from size distribution at delay $t_{2}=100 \mathrm{fs}$. Low energy edge of the $2 \mathrm{DE}$ spectrum at delays (b) $t_{2}=40 \mathrm{fs}$ and (c) $100 \mathrm{fs}$. The dynamics of the ESA signal (blue) requires the existence of a manifold of biexciton states.

ESA feature visible at $t_{2}=40 \mathrm{fs}$ is observed to disappear within the first $100 \mathrm{fs}$ of population times. We note that this ESA feature is not observed in the TA spectra upon band-edge pumping due to insufficient temporal and energy resolution. In the previous work by our group, we showed how this feature reflected dynamics within the exciton manifold $\mathrm{X}_{1}$ coupled to multiple biexciton states. At $t_{2}=40 \mathrm{fs}$, the ESA signal at $\left(E_{1}, E_{3}\right)=(1.92 \mathrm{eV}, 1.82 \mathrm{eV})$ requires a binding energy of $\Delta_{\mathrm{Abs}}\left(\mathrm{X}_{1} \mathrm{X}_{1}\right) \sim 65 \mathrm{meV}$. At $t_{2}=100 \mathrm{fs}$, this signal quickly disappears, consistent with a shift of the binding energy to lower values $\Delta_{\mathrm{Abs}}\left(\mathrm{X}_{1} \mathrm{X}_{1}\right) \sim 10 \mathrm{meV}$. This shift results from exciton relaxation between states which couple to different biexciton states.

Similarly to TA, signatures of the biexciton can also be observed by increasing the excitation intensity, thus directly creating biexciton populations. The biexciton population creates additional contribution to the signal, namely, SE back to X and ESA to TX. These contributions are superposed to the $2 \mathrm{D}$ spectrum and are the source of intensity dependant shifts visible in Figs. 11(c)-11(f). The series of fluence dependant $2 \mathrm{D}$ spectra represent a measurement of the spectral response as a function of a total of 4 parameters: $E_{1}, t_{2}, E_{3}$, and $I_{0}$. It is necessary to perform data reduction in order to analyze this dataset.

The global behavior of the 2D spectra can be analyzed by using the coordinates of the band-edge peak. The coordinates of the peak are reported in Fig. 13(a), and the peak signal level is reported in

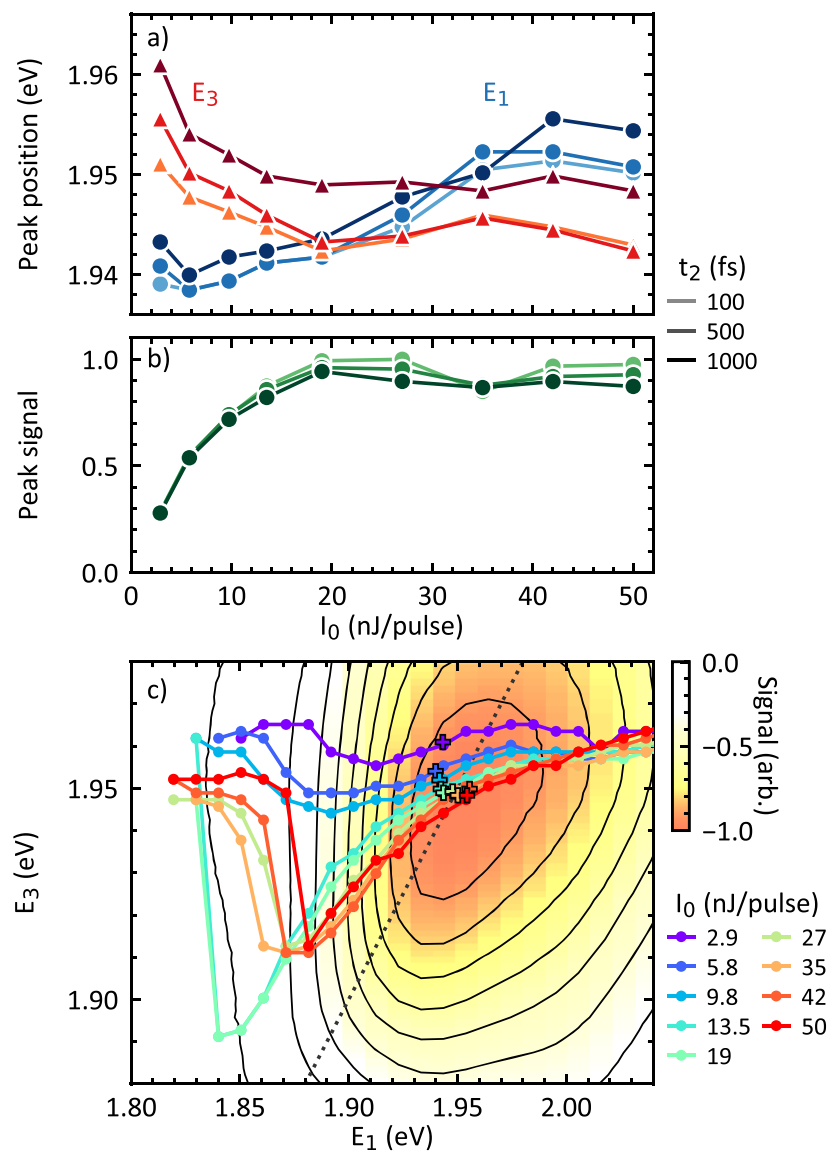

FIG. 13. The 2D spectra shift as a function of intensity. (a) The 2D peak shifts with both power and time. The emission coordinate $E_{3}$ shifts to lower values with intensity, indicative of $\mathrm{SE}$ from $\mathrm{BX}$. The excitation coordinate $E_{1}$ shifts to higher energies with intensity, indicating two-photon absorption into above band-edge biexcitons such as $X_{1} X_{2}$. The intensity is reported per pulse although two pulses act as the pump. The total excitation intensity is, thus, $2 I_{0}$. (b) Saturation of the signal as a function of intensity. (c) The peak of the transient response can be evaluated for each excitation energy, yielding a peak-line. The peak lines for $t_{2}=1$ ps are shown. The global peak of the 2D spectrum is shown by crosses.

Fig. 13(b). The lightness of the curves indicate the pump-probe delay $t_{2}$. With increasing intensity, the peak shifts to lower values of $E_{3}$ (red). This behavior corresponds to the peak shift observed in SRPP [Fig. 10(a)]. It is due to an increase in the SE signal from $\mathrm{BX}$ and a reduction of the ESA $\left(\mathrm{X}_{1} \rightarrow \mathrm{X}_{1} \mathrm{X}_{1}\right)$. This trend tracks the saturation of the $2 \mathrm{D}$ signal. In contrast, TA is unable to resolve the location of the peak along $E_{1}$ (blue). With increasing $I_{0}$, the location of the peak along $E_{1}$ shifts to higher energies. We attribute this effect to direct absorption into higher states of the biexciton, such as mixed biexcitons (e.g., $\mathrm{X}_{1} \mathrm{X}_{2}$ ). Inspection of Figs. 11(e) and 11(f) indicates a large increase in the signal up to $E_{1}=2.05 \mathrm{eV}$. Although these extra contributions cannot be separated, this observation is a direct signature of the existence of a coarse structure to the biexciton. The shift toward higher energies of the absorption maximum with increasing excitation energies must arise from the biexciton structure. 
The analysis of the peak dynamics can be refined by locating the peak of every pseudo-TA slice at fixed $E_{1}$ (i.e., black dots in Fig. 12) and reporting its location along $E_{3}$. This results in peak-lines, shown in Fig. 13(c) for $t_{2}=1$ ps. Peak-lines and the related center-line are a common way to study the complicated lineshapes of $2 \mathrm{D}$ spectroscopy. The results are shown in Fig. 13(a) for increasing excitation intensity at $t_{2}=1 \mathrm{ps}$. As $I_{0}$ increases, the low energy edge of the peakline shifts down, parallel to the diagonal. This signal is similar to the observation of a shift to lower energies along $E_{3}$ discussed in the previous paragraph. This shift indicates an increased contribution from BX, as previously discussed. The peak-line is also influenced by size inhomogeneity and by contributions from $\mathrm{X}_{2}$. At very high intensities $\left(I_{0}>25 \mathrm{~nJ} /\right.$ pulse $)$, the peak-line develops an abrupt jump to $E_{3} \sim 1.95 \mathrm{eV}$. We assign this shift to an increase in signals arising from two-photon absorption, isolated on the low-energy edge along $E_{1}$.

We now take a closer look at the signal on the low energy side along $E_{1}$, below the abrupt change in the peak line. Up to fifth order, we count a total of 120 pathways, which can be detected using our phase cycling and phase matching conditions. However, the spectral region where $E_{1}<1.85 \mathrm{eV}$ contains exclusively signals like those depicted in Fig. 14(a) as single-photon absorption is negligible in this spectral region. In these pathways, the first pulse interacts three times with the material, preparing the system into a $\left|\mathrm{X}_{1}\right\rangle\left\langle\mathrm{X}_{1} \mathrm{X}_{1}\right|$ coherence. The signal is, thus, shifted to lower

a)
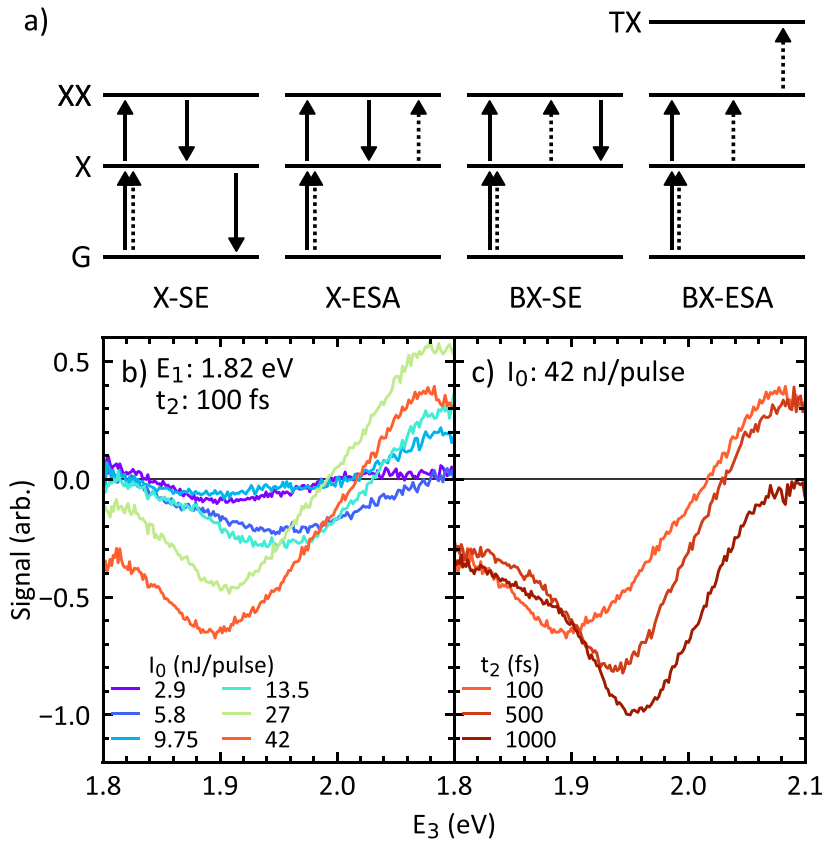

FIG. 14. Pseudo-TA spectra at low values of $E_{1}$ reveal dynamics in the finestructure states of the biexciton. (a) Diagrams corresponding to absorption at low values of $E_{1}$. Corresponding non-rephasing diagrams (not shown) also contribute. (b) Detuned excitation at $E_{1}=1.82 \mathrm{eV}$ isolates signals arising from two-photon absorption processes. At high intensity, the signal consists of SE from BX and ESA to TX. (c) Dynamics of this signal directly reveal dynamics in the $X_{1} X_{1}$ manifold. The apparent peak shift toward higher values of $E_{3}$ and signal decrease (more negative) is assigned to a reduction in BX-ESA due to hole dynamics. energies, at $E_{1}=E\left(\mathrm{X}_{1}\right)-\Delta_{\mathrm{Abs}}\left(\mathrm{X}_{1} \mathrm{X}_{1}\right)$. The second pulse interacts once with the material, projecting the coherence into a population either in X or BX. These populations then evolve during delay $t_{2}$. Finally, the third pulse probes these populations using the standard processes of SE and ESA. We note there are no contributions from GSB. Figure 14(a) represents four diagrams out of the 24 possible in this region. Only the rephasing diagrams are shown. The equivalent non-rephasing pathways can be obtained by changing the interactions of the first two pulses from the bra (solid) to the ket (dashed) and vice-versa. The other diagrams can be obtained by permuting the order of the interactions with the first pulse. All the 24 pathways are collected and contain identical population dynamics and transitions to one of the four shown here. It is also possible to construct diagrams with a single interaction with the first pulse and three interactions with the second pulse. However, these pathways evolve in a $|G\rangle\left\langle\mathrm{X}_{1}\right|$ coherence during delay $t_{1}$ and, thus, overlap with standard contributions along $E_{1}$. Finally, we note that double quantum coherences, formed by two interactions with both the first and second pulses, are not selected by our phase-cycling scheme. Up to fifth order, all signals located below the band-edge absorption appear in the same spectral locations as one of the four diagrams shown in Fig. 14(a).

The signals for two-photon excitation into the biexciton are shown in Figs. 14(b) and 14(c) for $E_{1}=1.82 \mathrm{eV}$. Intensity dependence of the signal is shown in Fig. 14(b). The signal arises from the combination of multiple contributions, with SE signals being negative and ESA signals being positive. This situation is similar to the description of Fig. 9. The signal is initially below the noise floor $\left(I_{0}<10 \mathrm{~nJ} /\right.$ pulse $)$. The signal clearly develops a derivative lineshape, dominated by SE for $E_{3}<2.0 \mathrm{eV}$ and ESA for $E_{3}>2.0 \mathrm{eV}$. This lineshape differs markedly from the lineshape for single-photon absorption. The use of a very low value of $E_{1}$ eliminates the possibility of excitation into mixed biexcitons, yielding pure signals from $\mathrm{X}_{1}$ and $\mathrm{X}_{1} \mathrm{X}_{1}$. The negative signal is dominated by the two SE pathways. The X-ESA signal is not clearly visible, indicating a large contribution from the BX pathways. The BX-ESA pathways gives rise to the positive signal at $E_{3}=2.05 \mathrm{eV}$.

The dynamics of the signals arising from two-photon absorption are shown in Fig. 14(c). The dynamics reveal a reduction of the ESA signal at $E_{3}>2.0 \mathrm{eV}$, accompanied by an apparent shift of the main SE signal to higher energies. We attribute these effects to dynamics in the fine structure manifold of the $\mathrm{X}_{1} \mathrm{X}_{1}$ biexciton. Dynamics in the $X_{1}$ exciton can be ruled out: dynamics of this magnitude are not present around the $\mathrm{X}_{1}$ peak at low intensity and are not observed by SRPP. ${ }^{24,80,106}$ The reduction in ESA indicates a shift to fine-structure states with a lower cross section for absorption into a P-like exciton. We attribute this effect to thermalization of the holes. The excitation into $\mathrm{X}_{1} \mathrm{X}_{1}$ generates holes in $1 \mathrm{~S}_{3 / 2}$-like states. The energy of the $1 \mathrm{P}_{3 / 2}$ hole level is located close to the $1 S_{3 / 2}$, within $40 \mathrm{meV}$. Thermalization processes can, thus, shift holes to the $1 \mathrm{P}_{3 / 2}$ manifold, reducing absorption probability into the triexciton. The reduction of ESA also contributes to the apparent shift in the SE signal. Indeed, hole thermalization should also reduce the intensity of the SE signal. The apparent intensity increase is, thus, assigned to the reduction in ESA. However, the signal at $t_{2}=100 \mathrm{fs}$ has a stronger SE intensity at $E_{3}=1.9 \mathrm{eV}$ and a different shape. We assign all these changes to dynamics in the $\mathrm{X}_{1} \mathrm{X}_{1}$ biexciton fine-structure manifold. The location of the spectroscopic features forming the curves 
in Fig. 14(c) can be estimated by locating the peak positions. The ESA to TX is located at $2.08 \mathrm{eV}$. Compared to the value obtained using t-PL, this yields an estimate of the width of the TX manifold $\delta_{\mathrm{TX}}=100 \mathrm{meV}$. The SE features are located at $1.90 \mathrm{eV}$ and $1.96 \mathrm{eV}$. Assuming the $\mathrm{SE}$ proceeds to different states of $\mathrm{X}_{1}$ separated by $\delta_{\mathrm{X}}$, these values allow an estimation of the width of the biexciton manifold of $20 \mathrm{meV}$, consistent with values obtained at low-fluence.

2DE spectroscopy is a powerful technique to probe the electronic response of materials, closely related to SRPP. It uses an interferometric pair of pulses to act as the pump, which resolves the time-dependent dynamics as a function of both excitation energy $E_{1}$, pump-probe delay $t_{2}$, and detection energy $E_{3}$. This enables increased time resolution ( $\sim 10 \mathrm{fs})$ and resolves effects arising from sample heterogeneity. Leveraging these advantages, we could observe a dynamical shift of the effective binding energy at low excitation intensity. This observation provided an estimate for the width of the biexciton manifold of $20 \mathrm{meV}$. At high excitation intensity, additional contributions arise from direct excitation into biexcitons via two-photon processes. Analyzing the response on the low energy side along $E_{1}$, we could directly observe dynamics in the finestructure manifold of $\mathrm{X}_{1} \mathrm{X}_{1}$ and ESA to $\mathrm{X}_{1} \mathrm{X}_{1} \mathrm{X}_{3}$ and, thus, estimate the width of both manifolds.

\section{DISCUSSION}

The experimental results probe the physics of confined multiexcitons on time scales ranging from $10 \mathrm{~s}$ of fs to $10 \mathrm{~s}$ of ns. By adjusting the excitation conditions, both excitation energy $E_{\text {ex }}$ and intensity $I_{0}$, multiple transitions are revealed. Quantifiable signals are recalled and summarized in Fig. 15 and Table I. The use of both emissive and absorptive techniques enables the study of different states: the a)

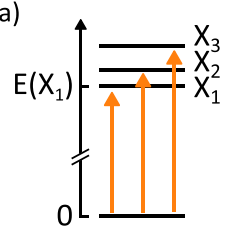

A B C

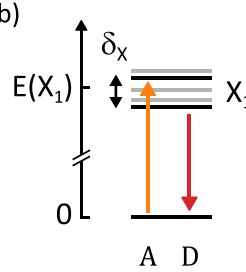

Abs SRPP 2DE tPL

c)

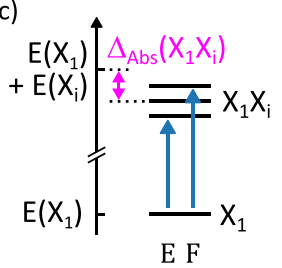

d)

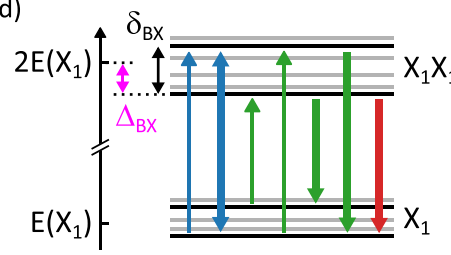

E G H I J K L e)

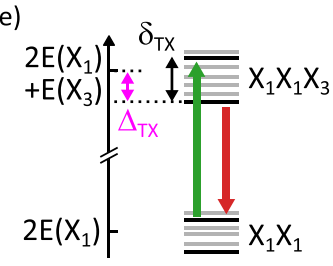

M N
FIG. 15. Summary of the observed transitions. The arrows are color-coded to indicate the experiment. Thin arrows refer to results from low excitation intensity, while wide arrows indicate high intensity. Capital letters refer to Table I. (a) Coarse structure of the exciton. (b) Fine structure of the band-edge exciton $X_{1}$. (c) Coarse structure of the biexciton. (d) Fine structure of the band-edge biexciton. (e) Fine structure of the lowest triexciton.
TABLE I. Summary of the observed transitions. Labels refer to Fig. 15.

\begin{tabular}{lcccc}
\hline \hline Label & Transition & Energy $(\mathrm{eV})$ & Exp. & Figures \\
\hline $\mathrm{A}$ & $\mathrm{G} \rightarrow \mathrm{X}_{1}$ & 1.934 & $\mathrm{Abs}$ & $3(\mathrm{c})$ \\
$\mathrm{B}$ & $\mathrm{G} \rightarrow \mathrm{X}_{2}$ & 2.002 & $\mathrm{Abs}$ & $3(\mathrm{c})$ \\
$\mathrm{C}$ & $\mathrm{G} \rightarrow \mathrm{X}_{3}$ & 2.180 & $\mathrm{Abs}$ & $3(\mathrm{c})$ \\
$\mathrm{D}$ & $\mathrm{X}_{1} \rightarrow \mathrm{G}$ & 1.892 & $\mathrm{PL}$ & $3(\mathrm{c})$ \\
$\mathrm{E}$ & $\mathrm{X}_{1} \rightarrow \mathrm{X}_{1} \mathrm{X}_{1}$ & 1.931 & $\mathrm{SRPP}$ & 9 \\
$\mathrm{~F}$ & $\mathrm{X}_{1} \rightarrow \mathrm{X}_{1} \mathrm{X}_{3}$ & 2.148 & $\mathrm{SRPP}$ & 9 \\
$\mathrm{G}$ & $\mathrm{X}_{1} \leftrightarrow \mathrm{X}_{1} \mathrm{X}_{1}$ & 1.929 & $\mathrm{SRPP}$ & 10 \\
$\mathrm{H}$ & $\mathrm{X}_{1} \rightarrow \mathrm{X}_{1}$ & $\sim 1.87$ & $2 \mathrm{DE}$ & $12(\mathrm{~b})$ \\
$\mathrm{I}$ & $\mathrm{X}_{1} \rightarrow \mathrm{X}_{1} \mathrm{X}_{1}$ & $>1.92$ & $2 \mathrm{DE}$ & $12(\mathrm{c})$ \\
$\mathrm{J}$ & $\mathrm{X}_{1} \mathrm{X}_{1} \rightarrow \mathrm{X}_{1}$ & 1.90 & $2 \mathrm{DE}$ & $14(\mathrm{~b})$ \\
$\mathrm{K}$ & $\mathrm{X}_{1} \mathrm{X}_{1} \rightarrow \mathrm{X}_{1}$ & 1.96 & $2 \mathrm{DE}$ & $14(\mathrm{c})$ \\
$\mathrm{L}$ & $\mathrm{X}_{1} \mathrm{X}_{1} \rightarrow \mathrm{X}_{1}$ & 1.877 & $\mathrm{t}-\mathrm{PL}$ & $6(\mathrm{c})$ \\
$\mathrm{M}$ & $\mathrm{X}_{1} \mathrm{X}_{1} \rightarrow \mathrm{X}_{1} \mathrm{X}_{1} \mathrm{X}_{3}$ & 2.08 & $2 \mathrm{DE}$ & $14(\mathrm{c})$ \\
$\mathrm{N}$ & $\mathrm{X}_{1} \mathrm{X}_{1} \mathrm{X}_{3} \rightarrow \mathrm{X}_{1} \mathrm{X}_{1}$ & 2.052 & $\mathrm{t}-\mathrm{PL}$ & $7(\mathrm{c})$ \\
\hline \hline
\end{tabular}

signal from emissive experiments is dominated by the lowest energy states, whereas absorptive experiments probe all states with a strong absorption cross section. The binding energies resulting from both absorptive and emissive experiments are recalled in Table II. The comparison of these results provides an estimate for the width of the exciton, biexciton, and triexciton manifold. The resulting values are shown in Table III. In addition, a number of qualitative observations revealing the presence of multiple BX and TX states are also reported. The results are summarized here.

The electronic structure of quantum confined excitons can readily be observed using linear absorption and PL spectroscopy. These observations are well established and well known. As they serve as a basis for the rest of the discussion, they are recalled here. The absorption spectrum reveals a series of excitons $\mathrm{X}_{i}$, which we refer to as the coarse structure of the exciton [Fig. 15(a)]. These exciton states are formed from the combination of different electron-hole pairs. Their transition energies upon absorption can be extracted from the absorption spectrum [Fig. 3(c)]. PL from X occurs at significantly lower energies than the absorption, which indicates the existence of a manifold of exciton states. The Stokes shift of the $\delta_{\mathrm{X}}$ exciton acts as an estimate for the width of the manifold of fine-structure states of the exciton [Fig. 15(b)].

The biexciton manifold can similarly be organized in a coarse structure and a fine structure. Hot excitons quickly relax to the band-edge exciton $\left(\mathrm{X}_{1}\right)$; thus, the biexcitons which can be formed by adding a second electron-hole pair to $\mathrm{X}_{1}$ are the easiest to observe. An analysis of the TA spectrum at low excitation intensity

TABLE II. Binding energies in absorptive and emissive measurements.

\begin{tabular}{lcc}
\hline \hline State & $\Delta_{\text {Abs }}(\mathrm{meV})$ & $\Delta_{\mathrm{Em}}(\mathrm{meV})$ \\
\hline $\mathrm{X}_{1} \mathrm{X}_{1}$ & 4.8 & 11 \\
$\mathrm{X}_{1} \mathrm{X}_{3}$ & 32 & \\
$\mathrm{X}_{1} \mathrm{X}_{1} \mathrm{X}_{3}$ & 105 & 128 \\
\hline \hline
\end{tabular}


TABLE III. Stokes shifts, establishing an estimate to the widths of fine-structure manifolds. All quantities are related to the lowest state of each manifold.

\begin{tabular}{ll}
\hline Quantity & Value (meV) \\
\hline$\delta_{\mathrm{X}}$ & 42 \\
$\delta_{\mathrm{BX}}$ & 54 \\
$\delta_{\mathrm{TX}}$ & 30 \\
\hline
\end{tabular}

enables the determination of binding energies for the mixed biexcitons $\Delta_{\mathrm{Abs}}\left(\mathrm{X}_{1} \mathrm{X}_{i}\right)$. Clear signatures for the $\mathrm{X}_{1} \rightarrow \mathrm{X}_{1} \mathrm{X}_{1}$ and $\mathrm{X}_{1} \rightarrow \mathrm{X}_{1} \mathrm{X}_{3}$ transitions can be observed, as shown in Fig. 15(c). The excitation from $\mathrm{X}_{1}$ to $\mathrm{X}_{1} \mathrm{X}_{2}$ is located in a crowded spectral region, and a precise determination of $\Delta_{\mathrm{Abs}}\left(\mathrm{X}_{1} \mathrm{X}_{2}\right)$ is, thus, difficult. The existence and rich structure of these mixed biexcitons is supported by $2 \mathrm{DE}$ spectroscopy at high excitation intensity. As excitation intensity increases, the $2 \mathrm{D}$ spectrum elongates toward higher values of $E_{1}$ (excitation energy). This effect can be observed qualitatively by comparing the $2 \mathrm{D}$ spectra at low and high intensities [Figs. 11(c)$11(\mathrm{f})]$ and quantified by reporting the coordinate of the $2 \mathrm{D}$ peak along $E_{1}$ [Fig. 13(a)]. These signatures directly reveal the biexciton manifold, but spectral congestion prevents their isolation. The width of the $\mathrm{X}$ and BX manifolds is comparable to the spacing between the $\mathrm{X}_{1}$ and $\mathrm{X}_{2}$ transitions. It is likely that the biexciton states formed of two $1 S_{e}$ electrons and two holes in the $1 S_{3 / 2}$ and $2 S_{3 / 2}$ states are strongly mixed and closely spaced. Furthermore, t-PL measurements indicate the presence of two recombination pathways for the band-edge TX: recombination of an S-like exciton $\left(\mathrm{X}_{1} \mathrm{X}_{1} \mathrm{X}_{3} \rightarrow \mathrm{X}_{1} \mathrm{X}_{3}\right)$ and recombination of a $\mathrm{P}$-like exciton $\left(\mathrm{X}_{1} \mathrm{X}_{1} \mathrm{X}_{3} \rightarrow \mathrm{X}_{1} \mathrm{X}_{1}\right)$ separated by $>170 \mathrm{meV}$.

The lowest biexciton, $\mathrm{X}_{1} \mathrm{X}_{1}$, can be studied in more details due to its longer lifetime and its spectral location. This enables the observation of multiple signals arising from the manifold of $\mathrm{X}_{1} \mathrm{X}_{1}$ fine-structure states, indicated in Fig. 15(d). The use of strong $\mathrm{X}_{1}$ excitation enabled the determination of an intensity independent effective binding energy $\Delta_{\mathrm{Abs}}\left(\mathrm{X}_{1} \mathrm{X}_{1}\right)=4.8 \mathrm{meV}$ (Fig. 10). The broad lineshapes of CdSe QDs complicate the analysis of SRPP due to the overlap of signatures and hole burning effects that are dependent on details of the spectrum of the excitation pulse. 2DE spectroscopy enables direct observation of these effects with increased time resolution. The dynamics at low and high intensities are reported in Table IV. At low $I_{0}$, the early time dynamics were shown to be inconsistent with either a single BX state or a fixed binding energy. The two ESA transitions are separated by $\sim 50 \mathrm{meV}$ (Fig. 12). The use of high excitation intensity enables the direct generation of biexcitons via two-photon processes. The signatures of biexciton populations were isolated from the $2 \mathrm{DE}$ spectra at low values of

TABLE IV. Dynamics reveal the structure of biexcitons. All binding energies were obtained using absorptive measurements for the band-edge biexciton.

\begin{tabular}{lccc}
\hline \hline$I_{0}(\mathrm{~nJ} / \mathrm{p})$ & $\Delta t(\mathrm{fs})$ & Early $(\mathrm{eV})$ & Late $(\mathrm{eV})$ \\
\hline 10 & 60 & 1.87 & 1.92 \\
42 & 900 & 1.90 & 1.96 \\
\hline \hline
\end{tabular}

$E_{1}$, revealing dynamics in the biexciton manifold [Fig. 14(c)]. The two SE transitions are separated by $\sim 60 \mathrm{meV}$. Similar two-photon absorption processes enable the observation of BX by t-PL. In this case, the band-edge biexciton state dominates the emission signals, with a lifetime $\tau_{\mathrm{BX}}=335$ ps. The emission from BX is located at slightly lower energy than emission from $\mathrm{X}$, yielding a value of $\Delta_{\mathrm{Em}}\left(\mathrm{X}_{1} \mathrm{X}_{1}\right)=11 \mathrm{meV}$ for the emissive states (Fig. 6). This result assumes that biexciton emission directly produces the emissive $\mathrm{X}_{1}$ state. Comparing the biexciton transitions observed by t-PL and SRPP yields a biexciton Stokes shift $\delta_{\mathrm{BX}}=54 \mathrm{meV}$. This value is consistent with the results from $2 \mathrm{DE}$ spectroscopy.

We observe the triexciton in both absorption and emission. The transitions are indicated in Fig. 15(e). The lowest optically active TX configuration has two S-like excitons $\left(\mathrm{X}_{1}\right)$ and one P-like exciton $\left(\mathrm{X}_{3}\right)$. This is due to filling of the doubly degenerate $1 \mathrm{~S}_{\mathrm{e}}$ electron state. Although these excitons are separated by $\sim 250 \mathrm{meV}$, their hole states are energetically close. ${ }^{4}$ ESA from BX to TX is clearly observed using 2DE at high excitation intensity (Fig. 14). PL corresponding to radiative TX recombination can be observed using $3.1 \mathrm{eV}$ excitation [Fig. 7(c)]. The recombination of a P-like exciton $\left(\mathrm{X}_{1} \mathrm{X}_{1} \mathrm{X}_{3} \rightarrow \mathrm{X}_{1} \mathrm{X}_{1}\right)$ is clearly separated from other emission pathways. A comparison of the transition energies observed in absorption and emission yield $\delta_{\mathrm{TX}}=30 \mathrm{meV}$. The emissive binding energy of the triexciton is $\Delta_{\mathrm{TX}}=139 \mathrm{meV}$. The lifetime of TX is very close to our experimental resolution, $\tau_{\mathrm{TX}}<100 \mathrm{ps}$. The binding energies of MX containing a mixture of S- and P-like excitons tend to be large. This is true both for the biexciton $\left(\mathrm{X}_{1} \mathrm{X}_{3}\right)$ and for the triexciton $\left(\mathrm{X}_{1} \mathrm{X}_{1} \mathrm{X}_{3}\right)$. We hypothesize this effect arises from a diminution of both the Coulomb and exchange interactions for states with different symmetries.

The results obtained by the use of t-PL, SRPP, and 2DE yield a consistent picture: the electronic spectrum of semiconductor nanocrystals exhibits a rich structure. Our results show that the biexciton and triexciton exhibit bound, bright states which result from stabilizing exciton-exciton interactions. Furthermore, each manifold consistently exhibits significant width. The experimental Stokes shifts $\delta$ provide an estimate for the widths of each manifold. Thus, the spectrum of states for $\mathrm{X}, \mathrm{BX}$, and TX covers a few tens of meV. For the biexciton, this value is larger than the binding energy: there are a significant number of states for which the exciton-exciton interaction is unfavorable. The width of the BX and TX manifolds, thus, reveals the limit of the concept of binding energy. The concept of binding energy arises in the context of bulk semiconductors. It seems reasonable when only a subset of the states can be observed as is the case when using PL. However, the binding energy is not uniquely defined for a complicated spectrum of discrete states as is the case in CdSe QDs. The use of absorptive experiments (SRPP and 2DE) simultaneously reveals multiple states. It is, thus, difficult to define a binding energy from absorptive experiments. This effect is exacerbated by dynamics, resulting in a time-dependent effective binding energy. ${ }^{106}$ The concept of binding energy is not entirely appropriate to describe the complicated electronic structure arising from quantum confinement.

The electronic structure of QDs would be fully addressed by resolving the individual eigenstates. This is not possible in the current case as the spacing between the states is narrower than the width of the individual lines. Inhomogeneous broadening arising from size distribution is the dominant line broadening mechanism in these 
materials. Its mitigation should enable the direct observation of the electronic structure. In principle, 2DE spectroscopy can separate static inhomogeneous broadening from other contributions. ${ }^{62,105}$ Although it succeeds in part at room temperature, significant spectral congestion remains. $29,30,32,33,104,107$ The recent low-temperature 2D experiment reports impressive line narrowing which should, in principle, reveal fine structure states. ${ }^{108,109}$ Another common approach consists in isolating the response from single nanocrystals. $23,84,85,110-113$ Performing a spatially resolved version of the current experiments should enable the observation of the electronic structure in the absence of ensemble broadening. ${ }^{114-116}$ Finally, the use of $2 \mathrm{Q}$ spectroscopy should enable the study of signals arising from the direct two-photon absorption without contributions from the single-photon absorption spectrum. ${ }^{117-119}$

\section{CONCLUSION}

In conclusion, we have analyzed the electronic structure of strongly confined semiconductor quantum dots with the help of time-resolved photoluminescence, state-resolved pump-probe, and two-dimensional electronic spectroscopies at varying excitation energies and intensities. The observations, performed over time scales spanning 6 orders of magnitude, reveal the spectrum of excitons and multiexcitons (biexcitons and triexcitons) arising from the interplay of exciton-nuclei interaction and exciton-exciton interactions. In strongly confined semiconductor QDs, the electronic structure of multiexcitons consists of multiple states spread over tens of meV. The range spanned by each manifold is at least comparable to the effective binding energies of the particles, stressing the limits of the concept of binding energy in these materials. The demonstrated techniques, analyses, and physics are generally applicable to electronic nanostructures and low-dimensional materials.

\section{ACKNOWLEDGMENTS}

This work was supported by the Canadian Foundation for Innovation (CFI), Natural Sciences and Engineering Research Council of Canada (NSERC), and McGill University. H.S. acknowledges support from SNSF. S.P. acknowledges support from FQRNT and NSERC.

\section{REFERENCES}

${ }^{1}$ A. I. Ekimov and A. A. Onushchenko, "Quantum size effect in the optical spectra of semiconductor microcrystals,” Sov. Phy. Semicon.-USSR 16, 775-778 (1982).

${ }^{2}$ M. G. Bawendi, W. L. Wilson, L. Rothberg, P. J. Carroll, T. M. Jedju, M. L. Steigerwald, and L. E. Brus, "Electronic structure and photoexcited-carrier dynamics in nanometer-size CdSe clusters,” Phys. Rev. Lett. 65, 1623-1626 (1990).

${ }^{3}$ D. J. Norris and M. G. Bawendi, "Measurement and assignment of the sizedependent optical spectrum in CdSe quantum dots," Phys. Rev. B 53, 16338-16346 (1996).

${ }^{4}$ A. L. Efros and M. Rosen, "The electronic structure of semiconductor nanocrystals,” Annu. Rev. Mater. Sci. 30, 475-521 (2000).

${ }^{5}$ L. Besombes, K. Kheng, and D. Martrou, "Exciton and biexciton fine structure in single elongated islands grown on a vicinal surface," Phys. Rev. Lett. 85, 425-428 (2000).

${ }^{6}$ M. V. Kovalenko, L. Manna, A. Cabot, Z. Hens, D. V. Talapin, C. R. Kagan, V. I. Klimov, A. L. Rogach, P. Reiss, D. J. Milliron, P. Guyot-Sionnnest, G. Konstantatos, W. J. Parak, T. Hyeon, B. A. Korgel, C. B. Murray, and W. Heiss, "Prospects of nanoscience with nanocrystals," ACS Nano 9, 1012-1057 (2015).
${ }^{7} \mathrm{P}$. Kambhampati, "Unraveling the structure and dynamics of excitons in semiconductor quantum dots," Acc. Chem. Res. 44, 1-13 (2011).

${ }^{8}$ M. Achermann, J. A. Hollingsworth, and V. I. Klimov, "Multiexcitons confined within a subexcitonic volume: Spectroscopic and dynamical signatures of neutral and charged biexcitons in ultrasmall semiconductor nanocrystals," Phys. Rev. B 68, 245302 (2003).

${ }^{9}$ A. V. Rodina and A. L. Efros, "Band-edge biexciton in nanocrystals of semiconductors with a degenerate valence band," Phys. Rev. B 82, 125324 (2010).

${ }^{10}$ V. I. Klimov, "Spectral and dynamical properties of multiexcitons in semiconductor nanocrystals,” Annu. Rev. Phys. Chem. 58, 635-673 (2007).

${ }^{11}$ C. R. Kagan, E. Lifshitz, E. H. Sargent, and D. V. Talapin, "Building devices from colloidal quantum dots," Science 353, aac5523 (2016).

${ }^{12}$ J. M. Pietryga, Y.-S. Park, J. Lim, A. F. Fidler, W. K. Bae, S. Brovelli, and V. I. Klimov, "Spectroscopic and device aspects of nanocrystal quantum dots," Chem. Rev. 116, 10513-10622 (2016).

${ }^{13}$ Y. Shirasaki, G. J. Supran, W. A. Tisdale, and V. Bulović, "Origin of efficiency roll-off in colloidal quantum-dot light-emitting diodes," Phys. Rev. Lett. 110, 217403 (2013).

${ }^{14}$ P. Kambhampati, "Multiexcitons in semiconductor nanocrystals: A platform for optoelectronics at high carrier concentration," J. Phys. Chem. Lett. 3, 1182-1190 (2012).

${ }^{15}$ J. I. Saari, M. M. Krause, B. R. Walsh, and P. Kambhampati, "Terahertz bandwidth all-optical modulation and logic using multiexcitons in semiconductor nanocrystals," Nano Lett. 13, 722-727 (2013).

${ }^{16}$ V. I. Klimov, D. W. McBranch, C. A. Leatherdale, and M. G. Bawendi, "Electron and hole relaxation pathways in semiconductor quantum dots," Phys. Rev. B 60, 13740-13749 (1999).

${ }^{17}$ V. I. Klimov, "Optical nonlinearities and ultrafast carrier dynamics in semiconductor nanocrystals,” J. Phys. Chem. B 104, 6112-6123 (2000).

${ }^{18}$ G. Nair, J. Zhao, and M. G. Bawendi, "Biexciton quantum yield of single semiconductor nanocrystals from photon statistics," Nano Lett. 11, 1136-1140 (2011).

${ }^{19}$ J. Shumway, A. Franceschetti, and A. Zunger, "Correlation versus mean-field contributions to excitons, multiexcitons, and charging energies in semiconductor quantum dots," Phys. Rev. B 63, 155316 (2001).

${ }^{20} \mathrm{M}$. Korkusinski, O. Voznyy, and P. Hawrylak, "Fine structure and size dependence of exciton and biexciton optical spectra in CdSe nanocrystals," Phys. Rev. B 82, 245304 (2010).

${ }^{21}$ Y. Z. Hu, S. W. Koch, M. Lindberg, N. Peyghambarian, E. L. Pollock, and F. F. Abraham, "Biexcitons in semiconductor quantum dots," Phys. Rev. Lett. 64, 1805-1807 (1990).

${ }^{22}$ H. Utzat, K. E. Shulenberger, O. B. Achorn, M. Nasilowski, T. S. Sinclair, and M. G. Bawendi, "Probing linewidths and biexciton quantum yields of single cesium lead halide nanocrystals in solution," Nano Lett. 17, 6838-6846 (2017).

${ }^{23}$ K. E. Shulenberger, T. S. Bischof, J. R. Caram, H. Utzat, I. Coropceanu, L. Nienhaus, and M. G. Bawendi, "Multiexciton lifetimes reveal triexciton emission pathway in CdSe nanocrystals," Nano Lett. 18, 5153 (2018).

${ }^{24}$ G. Grimaldi, J. J. Geuchies, W. van der Stam, I. du Fossé, B. Brynjarsson, N. Kirkwood, S. Kinge, L. D. Siebbeles, and A. J. Houtepen, "Spectroscopic evidence for the contribution of holes to the bleach of Cd-chalcogenide quantum dots," Nano Lett. 19, 3002-3010 (2019).

${ }^{25}$ S. L. Sewall, R. R. Cooney, K. E. H. Anderson, E. A. Dias, D. M. Sagar, and P. Kambhampati, "State-resolved studies of biexcitons and surface trapping dynamics in semiconductor quantum dots," J. Chem. Phys. 129, 084701 (2008).

${ }^{26}$ R. R. Cooney, S. L. Sewall, D. M. Sagar, and P. Kambhampati, "State-resolved manipulations of optical gain in semiconductor quantum dots: Size universality, gain tailoring, and surface effects," J. Chem. Phys. 131, 164706 (2009).

${ }^{27}$ S. L. Sewall, R. R. Cooney, E. A. Dias, P. Tyagi, and P. Kambhampati, "Stateresolved observation in real time of the structural dynamics of multiexcitons in semiconductor nanocrystals," Phys. Rev. B 84, 235304 (2011).

${ }^{28}$ K. W. Stone, D. B. Turner, K. Gundogdu, S. T. Cundiff, and K. A. Nelson, "Exciton-Exciton correlations revealed by two-quantum, two-dimensional fourier transform optical spectroscopy," Acc. Chem. Res. 42, 1452 (2009). 
${ }^{29}$ D. B. Turner, Y. Hassan, and G. D. Scholes, "Exciton superposition states in CdSe nanocrystals measured using broadband two-dimensional electronic spectroscopy," Nano Lett. 12, 880-886 (2012).

${ }^{30}$ T. A. Gellen, J. Lem, and D. B. Turner, "Probing homogeneous line broadening in CdSe nanocrystals using multidimensional electronic spectroscopy," Nano Lett. 17, 2809-2815 (2017).

${ }^{31}$ J. R. Caram, H. Zheng, P. D. Dahlberg, B. S. Rolczynski, G. B. Griffin, D. S. Dolzhnikov, D. V. Talapin, and G. S. Engel, "Exploring size and state dynamics in CdSe quantum dots using two-dimensional electronic spectroscopy," J. Chem. Phys. 140, 084701 (2014).

${ }^{32}$ H. Seiler, S. Palato, C. Sonnichsen, H. Baker, and P. Kambhampati, "Seeing multiexcitons through sample inhomogeneity: Band-edge biexciton structure in CdSe nanocrystals revealed by two-dimensional electronic spectroscopy," Nano Lett. 18, 2999-3006 (2018).

${ }^{33}$ H. Seiler, S. Palato, and P. Kambhampati, "Investigating exciton structure and dynamics in colloidal CdSe quantum dots with two-dimensional electronic spectroscopy," J. Chem. Phys. 149, 074702 (2018).

${ }^{34}$ K. E. Shulenberger, M. N. Ashner, S. K. Ha, F. Krieg, M. V. Kovalenko, W. A. Tisdale, and M. G. Bawendi, "Setting an upper bound to the biexciton binding energy in $\mathrm{CsPbBr}_{3}$ perovskite nanocrystals," J. Phys. Chem. Lett. 10, 5680-5686 (2019).

${ }^{35}$ M. N. Ashner, K. E. Shulenberger, F. Krieg, E. R. Powers, M. V. Kovalenko, M. G. Bawendi, and W. A. Tisdale, "Size-dependent biexciton spectrum in $\mathrm{CsPbBr}_{3}$ perovskite nanocrystals," ACS Energy Lett. 4, 2639-2645 (2019).

${ }^{36}$ J. A. Castañeda, G. Nagamine, E. Yassitepe, L. G. Bonato, O. Voznyy, S. Hoogland, A. F. Nogueira, E. H. Sargent, C. H. Cruz, and L. A. Padilha, "Efficient biexciton interaction in perovskite quantum dots under weak and strong confinement," ACS Nano 10, 8603-8609 (2016).

${ }^{37}$ D. Shi, V. Adinolfi, R. Comin, M. Yuan, E. Alarousu, A. Buin, Y. Chen, S. Hoogland, A. Rothenberger, K. Katsiev, Y. Losovyj, X. Zhang, P. A. Dowben, O. F. Mohammed, E. H. Sargent, and O. M. Bakr, "Low trap-state density and long carrier diffusion in organolead trihalide perovskite single crystals," Science 347, 519-522 (2015).

${ }^{38}$ H. Seiler, S. Palato, C. Sonnichsen, H. Baker, E. Socie, D. P. Strandell, and P. Kambhampati, "Two-dimensional electronic spectroscopy reveals liquid-like lineshape dynamics in $\mathrm{CsPbI}_{3}$ perovskite nanocrystals," Nat. Commun. 10, 4962 (2019).

${ }^{39}$ J. M. Richter, F. Branchi, F. V. d. A. Camargo, B. Zhao, R. H. Friend, G. Cerullo, and F. Deschler, "Ultrafast carrier thermalization in lead iodide perovskite probed with two-dimensional electronic spectroscopy," Nat. Commun. 8, 376 (2017).

${ }^{40}$ M. B. Price, J. Butkus, T. C. Jellicoe, A. Sadhanala, A. Briane, J. E. Halpert, K. Broch, J. M. Hodgkiss, R. H. Friend, and F. Deschler, "Hot-carrier cooling and photoinduced refractive index changes in organic-inorganic lead halide perovskites," Nat. Commun. 6, 8420 (2015).

${ }^{41}$ H. Zhu, K. Miyata, Y. Fu, J. Wang, P. P. Joshi, D. Niesner, K. W. Williams, $\mathrm{S}$. Jin, and X. Y. Zhu, "Screening in crystalline liquids protects energetic carriers in hybrid perovskites," Science 353, 1409-1413 (2016).

${ }^{42}$ G. Wang, E. Palleau, T. Amand, S. Tongay, X. Marie, and B. Urbaszek, "Polarization and time-resolved photoluminescence spectroscopy of excitons in $\mathrm{MoSe}_{2}$ monolayers," Appl. Phys. Lett. 106, 112101 (2015).

${ }^{43}$ X. X. Zhang, Y. You, S. Y. F. Zhao, and T. F. Heinz, "Experimental evidence for dark excitons in monolayer WSe ${ }_{2}$," Phys. Rev. Lett. 115, 257403 (2015).

${ }^{44}$ E. J. Sie, A. Steinhoff, C. Gies, C. H. Lui, Q. Ma, M. Rösner, G. Schönhoff, F. Jahnke, T. O. Wehling, Y. H. Lee, J. Kong, P. Jarillo-Herrero, and N. Gedik, "Observation of exciton redshift-blueshift crossover in monolayer WS2," Nano Lett. 17, 4210-4216 (2017).

${ }^{45}$ C. Ruppert, A. Chernikov, H. M. Hill, A. F. Rigosi, and T. F. Heinz, "The role of electronic and phononic excitation in the optical response of monolayer $\mathrm{WS}_{2}$ after ultrafast excitation," Nano Lett. 17, 644-651 (2017).

${ }^{46}$ G. Moody, C. Kavir Dass, K. Hao, C.-H. Chen, L.-J. Li, A. Singh, K. Tran, G. Clark, X. Xu, G. Berghäuser, E. Malic, A. Knorr, and X. Li, "Intrinsic homogeneous linewidth and broadening mechanisms of excitons in monolayer transition metal dichalcogenides," Nat. Commun. 6, 8315 (2015).

${ }^{47}$ K. J. Czech, B. J. Thompson, S. Kain, Q. Ding, M. J. Shearer, R. J. Hamers, S. Jin, and J. C. Wright, "Measurement of ultrafast excitonic dynamics of few-layer
$\mathrm{MoS}_{2}$ Using state-selective coherent multidimensional spectroscopy," ACS Nano 9, 12146-12157 (2015).

${ }^{48}$ A. Steinhoff, M. Florian, A. Singh, K. Tran, M. Kolarczik, S. Helmrich, A. W. Achtstein, U. Woggon, N. Owschimikow, F. Jahnke, and X. Li, "Biexciton fine structure in monolayer transition metal dichalcogenides," Nat. Phys. 14, 1199-1204 (2018).

${ }^{49}$ K. Hao, J. F. Specht, P. Nagler, L. Xu, K. Tran, A. Singh, C. K. Dass, C. Schüller, T. Korn, M. Richter, A. Knorr, X. Li, and G. Moody, "Neutral and charged intervalley biexcitons in monolayer $\mathrm{MoSe}_{2}$," Nat. Commun. 8, 15552 (2017).

${ }^{50}$ V. N. Soloviev, A. Eichhöfer, D. Fenske, and U. Banin, "Size-dependent optical spectroscopy of a homologous series of CdSe cluster molecules," J. Am. Chem. Soc. 123, 2354-2364 (2001).

${ }^{51}$ L. Jethi, T. G. Mack, and P. Kambhampati, "Extending semiconductor nanocrystals from the quantum dot regime to the molecular regime," J. Phys. Chem. C 121, 26102 (2017).

${ }^{52} \mathrm{O}$. V. Prezhdo, "Photoinduced dynamics in semiconductor quantum dots: Insights from time-domain ab initio studies," Acc. Chem. Res. 42, 2005-2016 (2009).

${ }^{53}$ K. E. Knowles, E. A. McArthur, and E. A. Weiss, "A multi-timescale map of radiative and nonradiative decay pathways for excitons in CdSe quantum dots," ACS Nano 5, 2026-2035 (2011).

${ }^{54}$ G. Rainò, I. Moreels, A. Hassinen, T. Stöferle, Z. Hens, and R. F. Mahrt, "Exciton dynamics within the band-edge manifold states: The onset of an acoustic phonon bottleneck," Nano Lett. 12, 5224-5229 (2012).

${ }^{55}$ A. Franceschetti, H. Fu, L. W. Wang, and A. Zunger, "Many-body pseudopotential theory of excitons in InP and CdSe quantum dots," Phys. Rev. B 60, 1819-1829 (1999).

${ }^{56}$ R. Osovsky, D. Cheskis, V. Kloper, A. Sashchiuk, M. Kroner, and E. Lifshitz, "Continuous-wave pumping of multiexciton bands in the photoluminescence spectrum of a single CdTe-CdSe core-shell colloidal quantum dot," Phys. Rev. Lett. 102, 197401 (2009).

${ }^{57}$ M. Califano, A. Franceschetti, and A. Zunger, "Lifetime and polarization of the radiative decay of excitons, biexcitons, and trions in CdSe nanocrystal quantum dots," Phys. Rev. B 75, 115401 (2007).

${ }^{58}$ B. P. Fingerhut, M. Richter, J.-W. Luo, A. Zunger, and S. Mukamel, "Dissecting biexciton wave functions of self-assembled quantum dots by double-quantumcoherence optical spectroscopy," Phys. Rev. B 86, 235303 (2012).

${ }^{59}$ J.-M. Caruge, Y. Chan, V. Sundar, H. J. Eisler, and M. G. Bawendi, "Transient photoluminescence and simultaneous amplified spontaneous emission from multiexciton states in CdSe quantum dots," Phys. Rev. B 70, 085316 (2004).

${ }^{60}$ M. Nirmal, D. J. Norris, M. Kuno, M. G. Bawendi, A. L. Efros, and M. Rosen, "Observation of the "dark exciton" in CdSe quantum dots," Phys. Rev. Lett. 75, 3728-3731 (1995).

${ }^{61}$ P. C. Sercel and A. L. Efros, "Band-Edge exciton in CdSe and other II-VI and III-V compound semiconductor nanocrystals - revisited," Nano Lett. 18, 40614068 (2018).

${ }^{62}$ S. Mukamel, Oxford Series in Optical and Imaging Sciences (Oxford University Press, New York, NY, 1995), Vol. 6, pp. xviii-543.

${ }^{63}$ M. Cho, Two-dimensional Optical Spectroscopy (CRC Press, 2009), p. 385.

${ }^{64}$ J. L. McHale, Molecular Spectroscopy, 2nd ed. (CRC Press, 2017), pp. 1-18.

${ }^{65}$ E. J. Heller, The Semiclassical Way to Dynamics and Spectroscopy (Princeton University Press, 2018).

${ }^{66}$ A. Franceschetti and Y. Zhang, "Multiexciton absorption and multiple exciton generation in CdSe quantum dots," Phys. Rev. Lett. 100, 136805 (2008).

${ }^{67}$ L.-W. Wang and A. Zunger, "High-energy excitonic transitions in CdSe quantum dots,” J. Phys. Chem. B 102, 6449-6454 (1998).

${ }^{68}$ A. Zunger, "Pseudopotential theory of semiconductor quantum dots," Phys. Status Solidi B 224, 727-734 (2001).

${ }^{69} \mathrm{O}$. V. Prezhdo, "Multiple excitons and the electron-phonon bottleneck in semiconductor quantum dots: An ab initio perspective," Chem. Phys. Lett. 460, 1-9 (2008).

${ }^{70}$ P. Hawrylak, "Excitonic artificial atoms: Engineering optical properties of quantum dots," Phys. Rev. B 60, 5597-5608 (1999). 
${ }^{71}$ K. E. Anderson, S. L. Sewall, R. R. Cooney, and P. Kambhampati, "Noise analysis and noise reduction methods in kilohertz pump-probe experiments," Rev. Sci. Instrum. 78, 073101 (2007).

${ }^{72}$ H. Seiler, S. Palato, B. E. Schmidt, and P. Kambhampati, "Simple fiber-based solution for coherent multidimensional spectroscopy in the visible regime," Opt. Lett. 42, 643 (2017).

${ }^{73} \mathrm{R}$. Trebino, Frequency-Resolved Optical Gating: The Measurement of Ultrashort Laser Pulses (Springer US, Boston, MA, 2000).

${ }^{74}$ Z. Zhang, K. L. Wells, E. W. J. Hyland, and H.-S. Tan, "Phase-cycling schemes for pump-probe beam geometry two-dimensional electronic spectroscopy," Chem. Phys. Lett. 550, 156-161 (2012).

${ }^{75} \mathrm{H}$. X. Fu, L. W. Wang, and A. Zunger, "Comparison of the k.p and the direct diagonalization approaches for describing the electronic structure of quantum dots," Appl. Phys. Lett. 71, 3433-3435 (1997).

${ }^{76}$ A. Zunger, "On the Farsightedness (hyperopia) of the Standard k.p Model," Phys. Status Solidi A 190, 467-475 (2002).

${ }^{77}$ S. V. Kilina, D. S. Kilin, and O. V. Prezhdo, "Breaking the phonon bottleneck in $\mathrm{PbSe}$ and CdSe quantum dots: Time-domain density functional theory of charge carrier relaxation," ACS Nano 3, 93-99 (2009).

${ }^{78}$ A. M. Kelley, "Electron-phonon coupling in CdSe nanocrystals," J. Phys. Chem. Lett. 1, 1296-1300 (2010).

${ }^{79} \mathrm{M}$. de Jong, L. Seijo, A. Meijerink, and F. T. Rabouw, "Resolving the ambiguity in the relation between Stokes shift and Huang-Rhys parameter," Phys. Chem. Chem. Phys. 17, 16959-16969 (2015).

${ }^{80}$ C. Zhang, T. N. Do, X. Ong, Y. Chan, and H.-S. Tan, "Understanding the features in the ultrafast transient absorption spectra of CdSe quantum dots," Chem. Phys. 481, 157-164 (2016).

${ }^{81}$ S. Palato, H. Seiler, L. McGovern, T. G. Mack, L. Jethi, and P. Kambhampati, "Electron dynamics at the surface of semiconductor nanocrystals," J. Phys. Chem. C 121, 26519-26527 (2017).

${ }^{82}$ C. Bonati, M. B. Mohamed, D. Tonti, G. Zgrablic, S. Haacke, F. van Mourik, and M. Chergui, "Spectral and dynamical characterization of multiexcitons in colloidal CdSe semiconductor quantum dots," Phys. Rev. B 71, 205317 (2005).

${ }^{83}$ V. I. Klimov, A. A. Mikhailovsky, D. W. McBranch, C. A. Leatherdale, and M. G. Bawendi, "Mechanisms for intraband energy relaxation in semiconductor quantum dots: The role of electron-hole interactions," Phys. Rev. B 61, R13349-R13352 (2000).

${ }^{84}$ M. Nirmal, B. O. Dabbousi, M. G. Bawendi, J. J. Macklin, J. K. Trautman, T. D. Harris, and L. E. Brus, "Fluorescence intermittency in single cadmium selenide nanocrystals," Nature 383, 802-804 (1996).

${ }^{85}$ S. A. Empedocles, D. J. Norris, and M. G. Bawendi, "Photoluminescence spectroscopy of single CdSe nanocrystallite quantum dots," Phys. Rev. Lett. 77, 3873-3876 (1996).

${ }^{86}$ G. Nair, S. M. Geyer, L.-Y. Chang, and M. G. Bawendi, "Carrier multiplication yields in $\mathrm{PbS}$ and PbSe nanocrystals measured by transient photoluminescence," Phys. Rev. B 78, 125325 (2008).

${ }^{87}$ R. Cooney, S. Sewall, D. Sagar, and P. Kambhampati, "Gain control in semiconductor quantum dots via state-resolved optical pumping," Phys. Rev. Lett. 102, 127404 (2009).

${ }^{88}$ B. R. Walsh, J. I. Saari, M. M. Krause, T. G. Mack, R. Nick, S. Coe-Sullivan, and P. Kambhampati, "Interfacial electronic structure in graded shell nanocrystals dictates their performance for optical gain," J. Phys. Chem. C 120, 19409-19415 (2016).

${ }^{89}$ C. D. Sonnichsen, T. Kipp, X. Tang, and P. Kambhampati, "Efficient optical gain in CdSe/CdS dots-in-rods," ACS Photonics 6, 382-388 (2018).

${ }^{90}$ R. Cooney, S. Sewall, K. Anderson, E. Dias, and P. Kambhampati, "Breaking the phonon bottleneck for holes in semiconductor quantum dots," Phys. Rev. Lett. 98, 177403 (2007).

${ }^{91}$ S. L. Sewall, R. R. Cooney, K. E. H. Anderson, E. A. Dias, and P. Kambhampati, "State-to-state exciton dynamics in semiconductor quantum dots," Phys. Rev. B 74, 235328 (2006)

${ }^{92}$ A. J. Houtepen, Z. Hens, J. S. Owen, and I. Infante, "On the origin of surface traps in colloidal II-VI semiconductor nanocrystals," Chem. Mater. 29, 752-761 (2017).
${ }^{93}$ B. Li, P. J. Brosseau, D. P. Strandell, T. G. Mack, and P. Kambhampati, "Photophysical action spectra of emission from semiconductor nanocrystals reveal violations to the Vavilov rule behavior from hot carrier effects," J. Phys. Chem. C 123, 5092-5098 (2019).

${ }^{94}$ P. Tyagi, R. R. Cooney, S. L. Sewall, D. M. Sagar, J. I. Saari, and P. Kambhampati, "Controlling piezoelectric response in semiconductor quantum dots via impulsive charge localization," Nano Lett. 10, 3062-3067 (2010).

${ }^{95}$ B. R. Walsh, C. Sonnichsen, T. G. Mack, J. I. Saari, M. M. Krause, R. Nick, S. Coe-Sullivan, and P. Kambhampati, "Excited state phononic processes in semiconductor nanocrystals revealed by excitonic state-resolved pump/probe spectroscopy," J. Phys. Chem. C 123, 3868-3875 (2019).

${ }^{96} \mathrm{P}$. Hamm and M. Zanni, Concepts and Methods of $2 D$ Infrared Spectroscopy (Cambridge University Press, Cambridge, 2011), ISBN: 978-1-1070-0005-6, pp. 1-286.

${ }_{97}^{9}$ R. M. Hochstrasser, "Two-dimensional spectroscopy at infrared and optical frequencies,” Proc. Natl. Acad. Sci. U. S. A. 104, 14190-14196 (2007).

${ }^{98}$ S. T. Cundiff and S. Mukamel, "Optical multidimensional coherent spectroscopy,” Phys. Today 66(7), 44 (2013).

${ }^{99}$ M. K. Petti, J. P. Lomont, M. Maj, and M. T. Zanni, “Two-dimensional spectroscopy is being used to address core scientific questions in biology and materials science," J. Phys. Chem. B 122, 1771-1780 (2018).

${ }^{100}$ S.-H. Shim and M. T. Zanni, "How to turn your pump-probe instrument into a multidimensional spectrometer: 2D IR and vis spectroscopies via pulse shaping," Phys. Chem. Chem. Phys. 11, 748-761 (2009).

${ }^{101}$ F. D. Fuller and J. P. Ogilvie, "Experimental implementations of twodimensional fourier transform electronic spectroscopy," Annu. Rev. Phys. Chem. 66, 667-690 (2015).

${ }^{102}$ K. L. M. Lewis, J. A. Myers, F. Fuller, P. F. Tekavec, and J. P. Ogilvie, "Strategies for scatter removal in two-dimensional electronic spectroscopy in the pumpprobe geometry," in International Conference on Ultrafast Phenomena (OSA, Washington, D.C., 2010), p. ME30.

${ }^{103}$ D. M. Jonas, "Two-dimensional femtosecond spectroscopy," Annu. Rev. Phys, Chem. 54, 425-463 (2003).

${ }^{104}$ S. D. Park, D. Baranov, J. Ryu, B. Cho, A. Halder, S. Seifert, S. Vajda, and D. M. Jonas, "Bandgap inhomogeneity of a PbSe quantum dot ensemble from twodimensional spectroscopy and comparison to size inhomogeneity from electron microscopy," Nano Lett. 17, 762-771 (2017).

${ }^{105}$ M. E. Siemens, G. Moody, H. Li, A. D. Bristow, and S. T. Cundiff, "Resonance lineshapes in two-dimensional Fourier transform spectroscopy," Opt. Express 18, 17699-17708 (2010).

${ }^{106}$ S. L. Sewall, A. Franceschetti, R. R. Cooney, A. Zunger, and P. Kambhampati, "Direct observation of the structure of band-edge biexcitons in colloidal semiconductor CdSe quantum dots," Phys. Rev. B 80, 081310 (2009).

${ }^{107}$ T. N. Do, C. Zhang, X. Ong, J. Lian, Y. Chan, and H.-S. Tan, "Measuring the ultrafast spectral diffusion dynamics of colloidal CdSe nanomaterials," MRS Adv. 4, 1-7 (2019).

${ }^{108}$ A. Liu, D. B. Almeida, W. K. Bae, L. A. Padilha, and S. T. Cundiff, "Nonmarkovian exciton-phonon interactions in core-shell colloidal quantum dots at femtosecond timescales," Phys. Rev. Lett. 123, 057403 (2019).

${ }^{109}$ A. Liu, D. B. Almeida, W. K. Bae, L. A. Padilha, and S. T. Cundiff, "Simultaneous existence of confined and delocalized vibrational modes in colloidal quantum dots," J. Phys. Chem. Lett. 10, 6144-6150 (2019).

${ }^{110}$ G. Chilla, T. Kipp, T. Menke, D. Heitmann, M. Nikolic, A. Kornowski, S. Förster, H. Weller, and A. Frömsdorf, "Direct observation of confined acoustic phonons in the photoluminescence spectra of a single CdSe-CdS-ZnS core-shellshell nanocrystal," Phys. Rev. Lett. 100, 057403 (2008).

${ }^{111}$ J. Cui, A. P. Beyler, I. Coropceanu, L. Cleary, T. R. Avila, Y. Chen, J. M. Cordero, S. L. Heathcote, D. K. Harris, O. Chen, J. Cao, and M. G. Bawendi, "Evolution of the single-nanocrystal photoluminescence linewidth with size and shell: Implications for exciton-phonon coupling and the optimization of spectral linewidths," Nano Lett. 16, 289-296 (2016).

${ }^{112}$ G. Schlegel, J. Bohnenberger, I. Potapova, and A. Mews, "Fluorescence decay time of single semiconductor nanocrystals," Phys. Rev. Lett. 88, 137401 (2002).

${ }^{113}$ F. Koberling, A. Mews, and T. Basché, "Single-dot spectroscopy of CdS nanocrystals and CdS/HgS heterostructures," Phys. Rev. B 60, 1921-1927 (1999). 
${ }^{114}$ A. C. Jones, N. M. Kearns, M. Bohlmann Kunz, J. T. Flach, and M. T. Zanni, "Multidimensional spectroscopy on the microscale: Development of a multimodal imaging system incorporating 2D white-light spectroscopy, broadband transient absorption, and atomic force microscopy," J. Phys. Chem. A 123, 10824 (2019).

${ }^{115}$ V. Tiwari, Y. A. Matutes, A. T. Gardiner, T. L. C. Jansen, R. J. Cogdell, and J. P. Ogilvie, "Spatially-resolved fluorescence-detected two-dimensional electronic spectroscopy probes varying excitonic structure in photosynthetic bacteria," Nat. Commun. 9, 4219 (2018).

${ }^{116}$ M. Aeschlimann, T. Brixner, A. Fischer, C. Kramer, P. Melchior, W. Pfeiffer, C. Schneider, C. Struber, P. Tuchscherer, and D. V. Voronine, "Coherent twodimensional nanoscopy," Science 333, 1723-1726 (2011).
${ }^{117}$ S. Mueller, S. Draeger, X. Ma, M. Hensen, T. Kenneweg, W. Pfeiffer, and T. Brixner, "Fluorescence-detected two-quantum and one-quantum-twoquantum 2D electronic spectroscopy," J. Phys. Chem. Lett. 9, 1964-1969 (2018).

${ }^{118}$ S. Mueller, J. Lüttig, P. Malý, L. Ji, J. Han, M. Moos, T. B. Marder, U. H. Bunz, A. Dreuw, C. Lambert, and T. Brixner, "Rapid multiple-quantum threedimensional fluorescence spectroscopy disentangles quantum pathways," Nat. Commun. 10, 4735 (2019).

${ }^{119} \mathrm{H}$. Li, A. D. Bristow, M. E. Siemens, G. Moody, and S. T. Cundiff, "Unraveling quantum pathways using optical 3D Fourier-transform spectroscopy," Nat. Commun. 4, 1390 (2013). 